\title{
Total Synthesis of (-)-FD-838 and (-)-Cephalimysin A
}

\author{
Deokhee Jo ${ }^{\dagger}$ and Sunkyu Han ${ }^{* \dagger, *}$ \\ ${ }^{\dagger}$ Department of Chemistry, Korea Advanced Institute of Science and Technology (KAIST), Daejeon, 34141, \\ Republic of Korea \\ ${ }^{\star}$ Center for Catalytic Hydrocarbon Functionalizations, Institute for Basic Science (IBS), Daejeon, 34141, \\ Republic of Korea
}

\section{Supporting Information}

General Procedures $\mathrm{S} 3$

Materials and Instrumentations S3

Positional Numbering System $\mathrm{S} 4$

tert-Butyl 5-(5-ethylfuran-2-yl)-5-hydroxy-4-methyl-3-oxopentanoate (S1) S5

tert-Butyl 5-(5-ethylfuran-2-yl)-4-methyl-3,5-dioxopentanoate (19) S6

$\underline{(5 S, 6 S)-2,2,3,3,9,9,10,10-O c t a m e t h y l-5-p h e n y l-4,8-d i o x a-3,9-d i s i l a u n d e c a n-6-a m i n e ~(S 3) ~}$

(2S,3S)-2-Amino-3-((tert-butyldimethylsilyl)oxy)-3-phenylpropan-1-ol (17) S8

$\mathrm{N}$-((1S,2S)-1-((tert-Butyldimethylsilyl)oxy)-3-hydroxy-1-phenylpropan-2-yl)-5-(5ethylfuran-2-yl)-4-methyl-3,5-dioxopentanamide (20) S9

$(5 S, 8 S, 9 R)-8-((S)-(($ tert-Butyldimethylsilyl)oxy)(phenyl)methyl)-2-(5-ethylfuran-2-yl)-8,9dihydroxy-3-methyl-1-oxa-7-azaspiro[4.4]non-2-ene-4,6-dione $\quad(\mathbf{2 2}) \quad \& \quad(5 R, 8 R, 9 S)-8-((S)$ ((tert-butyldimethylsilyl)oxy)(phenyl)methyl)-2-(5-ethylfuran-2-yl)-8,9-dihydroxy-3-methyl1-oxa-7-azaspiro[4.4]non-2-ene-4,6-dione (23) S10

$\underline{(5 S, 8 S, 9 R)-8-((S)-((t e r t-B u t y l d i m e t h y l s i l y l) o x y)(\text { phenyl)methyl)-2-(5-ethylfuran-2-yl)-9- }}$ hydroxy-8-methoxy-3-methyl-1-oxa-7-azaspiro[4.4]non-2-ene-4,6-dione (30) $\quad$ S12

$(5 S, 8 R, 9 R)-2-(5-E t h y l f u r a n-2-y l)-9-h y d r o x y-8-((S)$-hydroxy(phenyl)methyl)-8-methoxy-3methyl-1-oxa-7-azaspiro[4.4]non-2-ene-4,6-dione (31) S13 $\underline{(-)-F D-838(5)}$ S14

tert-Butyl (E)-5-hydroxy-4-methyl-3-oxoundec-8-enoate (S4) S15 tert-Butyl (E)-4-methyl-3,5-dioxoundec-8-enoate (33) S16 $(E)-N$-((1S,2S)-1-((tert-Butyldimethylsilyl)oxy)-3-hydroxy-1-phenylpropan-2-yl)-4-methyl3,5-dioxoundec-8-enamide (34) S17

(5S,8S,9R)-8- $((S)-(($ tert-Butyldimethylsilyl)oxy $)($ phenyl $)$ methyl $)-2-((E)-h e x-3-e n-1-y l)-9-$ hydroxy-8-methoxy-3-methyl-1-oxa-7-azaspiro[4.4]non-2-ene-4,6-dione (36) $\quad$ S18 
$(5 S, 8 R, 9 R)-2-((E)-H e x-3-e n-1-y l)-9-h y d r o x y-8-((S)$-hydroxy(phenyl)methyl)-8-methoxy-3methyl-1-oxa-7-azaspiro[4.4]non-2-ene-4,6-dione (S5) S20

(-)-Cephalimysin A (6)

Table S1. Comparison of ${ }^{1} \mathrm{H}$ NMR Spectral Data of Our Synthetic (-)-FD-838 (5) with Isolation Literature S22

Table S2. Comparison of ${ }^{13}$ C NMR Spectral Data of Our Synthetic (-)-FD-838 (5) with Isolation Literature S23

Table S3. Comparison of ${ }^{1}$ H NMR Spectral Data of Our Synthetic (-)-Cephalimysin A (6) with Isolation Literature S24

Table S4. Comparison of ${ }^{13} \mathrm{C}$ NMR Spectral Data of Our Synthetic (-)-Cephalimysin A (6) with Isolation Literature S25

HPLC Traces of Synthetic (-)-FD-838, (+)-FD-838, and Their Mixture S26

Confirmation of the Absolute Stereochemistry of Our Synthetic (-)-FD-838 S27

Comparison of the CD Spectra of Our Synthetic (-)-Cephalimysin A and the Natural Sample S28 


\section{General Procedures}

All reactions were performed in oven-dried or flame-dried round-bottomed flasks. Unless otherwise noted, the flasks were fitted with rubber septa and reactions were conducted under a positive pressure of argon. Stainless steel syringes or cannulae were used to transfer air- and moisture-sensitive liquids. Flash column chromatography was performed as described by Still et al. using silica gel (60 $\AA$ pore size, $40-63 \mu \mathrm{m}, 4-6 \% \mathrm{H}_{2} \mathrm{O}$ content, Merck). ${ }^{1}$ Analytical thin-layer chromatography (TLC) was performed using glass plates pre-coated with $0.25 \mathrm{~mm}$ silica gel impregnated with a fluorescent indicator $(254 \mathrm{~nm})$. Thin layer chromatography plates were visualized by exposure to ultraviolet light and/or by exposure to an aqueous solution of ceric ammonium molybdate (CAM) or an aqueous solution of potassium permanganate (KMnO4).

\section{Materials and Instrumentations}

Unless otherwise stated, all commercial reagents and solvents were used without additional purification with the following exceptions: dichloromethane and tetrahydrofuran were purchased commercial vendors, respectively, and were purified by the method of Grubbs et al. under positive argon pressure. ${ }^{2}$

Proton and carbon nuclear magnetic resonance spectra were recorded with Bruker AVANCE III HD Nanobay (400 MHz), Bruker AVANCE III HD (400 MHz), or Agilent Technologies DD2 (600 MHz) spectrometers. Proton nuclear magnetic resonance spectra are referenced from the residual protium in the NMR solvent $\left(\mathrm{CDCl}_{3}: \delta 7.24\left(\mathrm{CHCl}_{3}\right)\right.$, benzene- $d_{6}$ : $\delta 7.16$ (benzene- $\left.d_{5}\right), \mathrm{CD}_{2} \mathrm{Cl}_{2}: \delta 5.32\left(\mathrm{CHDCl}_{2}\right)$. Data are reported in the following manners: chemical shift in ppm [multiplicity $(\mathrm{s}=$ singlet, $\mathrm{d}=$ doublet, $\mathrm{t}=$ triplet, $\mathrm{q}=$ quartet, $\mathrm{m}=$ multiplet, app = apparent, br = broad), coupling constant(s) in Hertz, integration]. Carbon-13 nuclear magnetic resonance spectra are referenced from the carbon resonances of the solvent $\left(\mathrm{CDCl}_{3}\right.$ : $\delta 77.23$, benzene- $\left.d_{6}: \delta 128.39, \mathrm{CD}_{2} \mathrm{Cl}_{2}: \delta 54.00\right)$. Data are reported in the following manners: chemical shift in ppm. High resolution mass spectra were obtained from KAIST Research Analysis Center (Daejeon) by using ESI method. Optical rotations were recorded with Jasco P-2000 Polarimeter equipped with a temperature controller.

\footnotetext{
${ }^{1}$ Still, W. C.; Kahn, M.; Mitra, A. Rapid chromatographic technique for preparative separations with moderate resolution. $J$. Org. Chem. 1978, 43, 2923.

${ }^{2}$ Pangborn, A. B.; Giardello, M. A.; Grubbs, R. H.; Rosen, R. K.; Timmers, F. J. Safe and Convenient Procedure for Solvent Purification. Organometallics, 1996, 15, 1518.
} 


\section{Positional Numbering System}
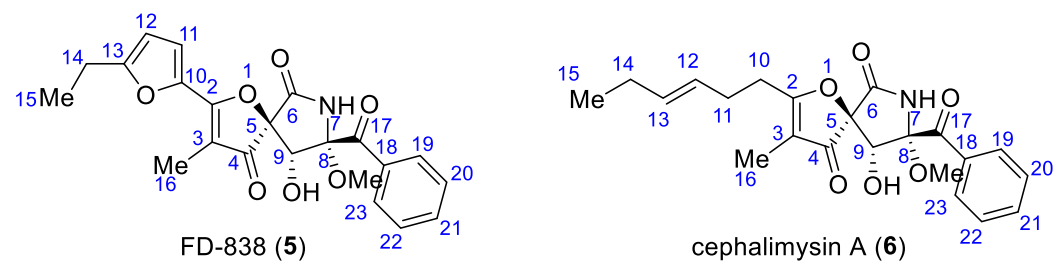


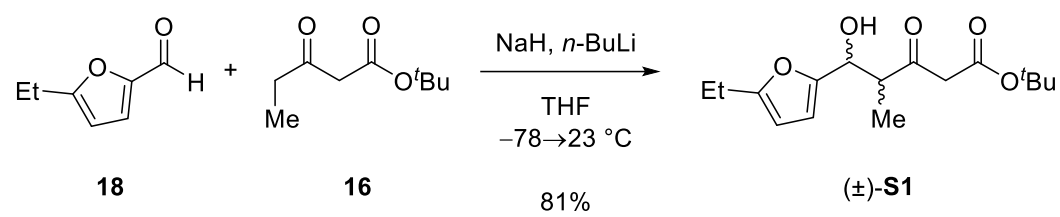

\section{tert-Butyl 5-(5-ethylfuran-2-yl)-5-hydroxy-4-methyl-3-oxopentanoate (S1):}

To a suspension of sodium hydride ( $\mathrm{NaH}, 60 \%$ dispersion in mineral oil, $352 \mathrm{mg}, 8.80$ mmol, 1.10 equiv) in tetrahydrofuran $(20 \mathrm{~mL})$ at $0{ }^{\circ} \mathrm{C}$ was added tert-butyl 3-oxopentanoate $(\mathbf{1 6}, 1.42 \mathrm{~mL}, 8.00 \mathrm{mmol}, 1$ equiv) dropwise under argon. After the reaction mixture was stirred at $0{ }^{\circ} \mathrm{C}$ for $30 \mathrm{~min}$, a solution of $n$-BuLi (2.50 M in $n$-hexane, $3.52 \mathrm{~mL}, 8.80 \mathrm{mmol}, 1.10$ equiv) was added dropwise to the reaction mixture. The reaction mixture was stirred at $0{ }^{\circ} \mathrm{C}$ for 30 $\mathrm{min}$. The resulting solution was cooled to $-78^{\circ} \mathrm{C}$. Subsequently, 5-ethylfuran-2-carbaldehyde $(\mathbf{1 8}, 961 \mu \mathrm{L}, 8.00 \mathrm{mmol}, 1.00$ equiv) in tetrahydrofuran $(20 \mathrm{~mL})$ was transferred by cannula to the reaction mixture. The reaction mixture was allowed to warm to $23{ }^{\circ} \mathrm{C}$. After $3 \mathrm{~h}$, saturated aqueous ammonium chloride solution $(30 \mathrm{~mL})$ was added to the reaction mixture, the resulting mixture was diluted with ethyl acetate $(60 \mathrm{~mL})$, and the layers were separated. The aqueous layer was extracted with ethyl acetate $(2 \times 60 \mathrm{~mL})$, and the combined organic layers were dried over anhydrous sodium sulfate and concentrated under reduced pressure. The resulting crude residue was purified by flash column chromatography (silica gel: diam. $5 \mathrm{~cm}$, ht. $9 \mathrm{~cm}$; eluent: ethyl acetate : hexanes $=1: 10$ to $1: 2)$ to afford $( \pm)$-S1 $(1: 1$ mixture of diastereomers, $1.91 \mathrm{~g}$, $81 \%$ ) as a pale yellow oil.

${ }^{1} \mathrm{H}$ NMR and ${ }^{13} \mathrm{C}$ NMR of S1 were taken with sample containing 1:1 mixture of inseparable diastereomers.

${ }^{1}$ H NMR $\left(400.1 \mathrm{MHz}, \mathrm{CDCl}_{3}\right) \delta$ 6.16-6.14 (m, 1H), 6.14-6.12 (m, 1H), 5.90-5.88 (m, 2H), $4.96(\mathrm{~d}, J=4.7 \mathrm{~Hz}, 1 \mathrm{H}), 4.69(\mathrm{~d}, J=8.6 \mathrm{~Hz}, 1 \mathrm{H}), 3.53-3.35(\mathrm{~m}, 4 \mathrm{H}), 3.27(\mathrm{dq}, J=8.6,7.1 \mathrm{~Hz}$, $1 \mathrm{H}), 3.14(\mathrm{qd}, J=7.1,4.8 \mathrm{~Hz}, 1 \mathrm{H}), 2.66(\mathrm{br}-\mathrm{s}, 2 \mathrm{H}), 2.65-2.55(\mathrm{~m}, 4 \mathrm{H}), 1.46(\mathrm{~s}, 9 \mathrm{H}), 1.45$ (s, $9 \mathrm{H}), 1.22-1.15(\mathrm{~m}, 9 \mathrm{H}), 0.98(\mathrm{~d}, J=7.1 \mathrm{~Hz}, 3 \mathrm{H})$.

${ }^{13}$ C NMR $\left(150.7 \mathrm{MHz}, \mathrm{CDCl}_{3}\right) \delta 207.4,206.8,166.6,166.5,158.2,157.6,152.2,152.2,108.8$, $107.8,104.8,104.7,82.4,82.3,70.5,68.7,51.2,50.8,50.7,49.9,28.2,28.2,21.6,21.5,14.0$, 12.3, 12.3, 11.3.

HRMS (ESI): Calculated for $\mathrm{C}_{16} \mathrm{H}_{24} \mathrm{O}_{5}[\mathrm{M}+\mathrm{Na}]^{+}: 319.1516$, found: 319.1515 .

TLC (ethyl acetate : hexanes = $1: 3$ ) Rf: 0.50 (CAM, UV). 


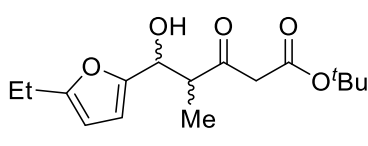

$( \pm)-S 1$

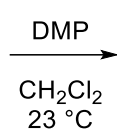

$69 \%$

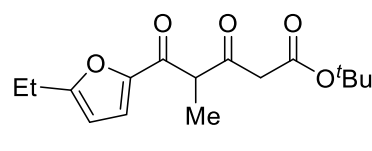

$( \pm)-19$

\section{tert-Butyl 5-(5-ethylfuran-2-yl)-4-methyl-3,5-dioxopentanoate (19):}

To a stirred solution of ( \pm )-S1 (735 mg, $2.48 \mathrm{mmol}, 1$ equiv) in dichloromethane (37 $\mathrm{mL}$ ) was added Dess-Martin periodinane (DMP, $1.58 \mathrm{~g}, 3.72 \mathrm{mmol}, 1.50$ equiv) at $23^{\circ} \mathrm{C}$. After $30 \mathrm{~min}$, a mixture of saturated aqueous sodium thiosulfate solution and saturated aqueous sodium bicarbonate solution $(1: 1,80 \mathrm{~mL})$ was added to the reaction mixture, and the layers were separated. The aqueous layer was extracted with dichloromethane $(2 \times 40 \mathrm{~mL})$, and the combined organic layers were dried over anhydrous sodium sulfate and concentrated under reduced pressure. The resulting crude residue was purified by flash column chromatography (silica gel: diam. $4 \mathrm{~cm}$, ht. $12 \mathrm{~cm}$; eluent: ethyl acetate : hexanes $=1: 10$ to $1: 4$ ) to afford $( \pm)$ 19 (506 $\mathrm{mg}, 69 \%)$ as a pale yellow oil.

${ }^{1} \mathbf{H}$ NMR $\left(400.1 \mathrm{MHz}, \mathrm{CDCl}_{3}\right) \delta 7.18(\mathrm{dt}, J=3.6,0.5 \mathrm{~Hz}, 1 \mathrm{H}), 6.16(\mathrm{dt}, J=3.6,0.9 \mathrm{~Hz}, 1 \mathrm{H})$, $4.36(\mathrm{q}, J=7.0 \mathrm{~Hz}, 1 \mathrm{H}), 3.46-3.34(\mathrm{~m}, 2 \mathrm{H}), 2.72-2.64(\mathrm{~m}, 2 \mathrm{H}), 1.37(\mathrm{~d}, J=7.0 \mathrm{~Hz}, 3 \mathrm{H}), 1.36$ $(\mathrm{s}, 9 \mathrm{H}), 1.23(\mathrm{t}, J=7.6 \mathrm{~Hz}, 3 \mathrm{H})$.

${ }^{13} \mathbf{C}$ NMR $\left(150.7 \mathrm{MHz}, \mathrm{CDCl}_{3}\right) \delta 199.5,184.7,166.2,164.3,150.4,120.9,108.2,82.2,55.8$, 49.0, 28.0, 21.9, 13.3, 11.8 .

HRMS (ESI): Calculated for $\mathrm{C}_{16} \mathrm{H}_{22} \mathrm{O}_{5}[\mathrm{M}+\mathrm{Na}]^{+}: 317.1359$, found: 317.1365 .

TLC (ethyl acetate : hexanes = $1: 4)$ Rf: $0.46($ CAM, UV). 


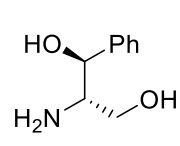

S2

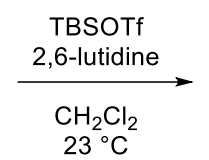

$98 \%$

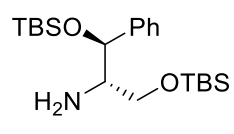

S3

\section{$\underline{(5 S, 6 S)-2,2,3,3,9,9,10,10-O c t a m e t h y l-5-p h e n y l-4,8-d i o x a-3,9-d i s i l a u n d e c a n-6-a m i n e ~(S 3): ~}$}

To a stirred solution of $\mathbf{S 2}(345 \mathrm{mg}, 2.00 \mathrm{mmol}, 1$ equiv) and 2,6-lutidine (1.18 mL, $10.0 \mathrm{mmol}, 5.00$ equiv) in dichloromethane $(24 \mathrm{~mL})$ was added tert-butyldimethylsilyl trifluoromethanesulfonate (TBSOTf, $1.87 \mathrm{~mL}, 8.00 \mathrm{mmol}, 4.00$ equiv) dropwise at $23{ }^{\circ} \mathrm{C}$. After $16 \mathrm{~h}$, saturated aqueous sodium bicarbonate solution $(30 \mathrm{~mL})$ was added to the reaction mixture, and the layers were separated. The aqueous layer was extracted with dichloromethane $(2 \times 30$ $\mathrm{mL}$ ), and the combined organic layers were dried over anhydrous sodium sulfate and concentrated under reduced pressure. The resulting crude residue was purified by flash column chromatography (silica gel: diam. $4 \mathrm{~cm}$, ht. $11 \mathrm{~cm}$; eluent: dichloromethane : methanol = 40:1 to $20: 1)$ to afford $\mathbf{S 3}$ (774 $\mathrm{mg}, 98 \%$ ) as a pale yellow oil.

${ }^{1} \mathbf{H}$ NMR $\left(400.1 \mathrm{MHz}, \mathrm{CDCl}_{3}\right) \delta 7.30-7.18(\mathrm{~m}, 5 \mathrm{H}), 4.71(\mathrm{~d}, J=5.2 \mathrm{~Hz}, 1 \mathrm{H}), 3.48(\mathrm{dd}, J=$ 9.9, $5.9 \mathrm{~Hz}, 1 \mathrm{H}), 3.36$ (dd, $J=9.9,5.1 \mathrm{~Hz}, 1 \mathrm{H}), 2.73$ (app-q, $J=5.4 \mathrm{~Hz}, 1 \mathrm{H}), 1.62$ (br-s, 2H), 0.89 (s, 9H), 0.87 (s, 9H), 0.02 (s, 3H), 0.01 (s, 3H), 0.01 (s, 3H), -0.22 (s, 3H).

${ }^{13}$ C NMR $\left(100.6 \mathrm{MHz}, \mathrm{CDCl}_{3}\right) \delta 143.3,128.2,127.4,126.7,75.3,64.1,60.2,26.1,26.1,18.4$, $18.4,-4.3,-4.9,-5.2,-5.2$.

HRMS (ESI): Calculated for $\mathrm{C}_{21} \mathrm{H}_{41} \mathrm{NO}_{2} \mathrm{Si}_{2}[\mathrm{M}+\mathrm{H}]^{+}:$396.2749, found: 396.2743 .

TLC (dichloromethane : methanol = 9 : 1) Rf: 0.69 (KMnO4).

$[\boldsymbol{\alpha}]_{\mathbf{D}^{25}}:+34.2\left(c 1.21, \mathrm{CHCl}_{3}\right)$. 


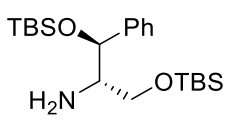

S3

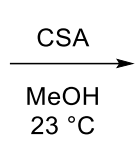

$92 \%$

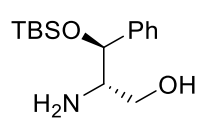

17

\section{(2S,3S)-2-Amino-3-((tert-butyldimethylsilyl)oxy)-3-phenylpropan-1-ol (17):}

To a stirred solution of $\mathbf{S 3}$ (774 mg, $1.96 \mathrm{mmol}, 1$ equiv) in methanol (98 mL) was added 10-camphorsulfonic acid (CSA, $2.29 \mathrm{~g}, 9.78 \mathrm{mmol}, 5.00$ equiv) at $23{ }^{\circ} \mathrm{C}$. After $18 \mathrm{~h}$, sodium bicarbonate $(2.00 \mathrm{~g})$ was added to the reaction mixture, and the volatile was removed under reduced pressure. Ethyl acetate $(30 \mathrm{~mL})$ and water $(30 \mathrm{~mL})$ were added to the resulting mixture, and the layers were separated. The aqueous layer was extracted with ethyl acetate (2 $\times 30 \mathrm{~mL}$ ), and the combined organic layers were dried over anhydrous sodium sulfate and concentrated under reduced pressure. The resulting crude residue was purified by flash column chromatography (silica gel: diam. $4 \mathrm{~cm}$, ht. $9 \mathrm{~cm}$; eluent: dichloromethane : methanol =10:1) to afford 17 (504 $\mathrm{mg}, 92 \%)$ as a pale yellow oil.

${ }^{1} \mathbf{H}$ NMR $\left(400.1 \mathrm{MHz}, \mathrm{CDCl}_{3}\right) \delta 7.32-7.21(\mathrm{~m}, 5 \mathrm{H}), 4.59(\mathrm{~d}, J=4.9 \mathrm{~Hz}, 1 \mathrm{H}), 3.48(\mathrm{dd}, J=$ 10.7, 4.5 Hz, 1H), 3.34 (dd, $J=10.7,7.2 \mathrm{~Hz}, 1 \mathrm{H}$ ), 2.85 (app-dt, $J=7.2,4.7 \mathrm{~Hz}, 1 \mathrm{H}$ ), 2.11 (br$\mathrm{s}, 3 \mathrm{H}), 0.87$ (s, 9H), 0.01 (s, 3H), -0.23 (s, 3H).

${ }^{13}$ C NMR $\left(100.6 \mathrm{MHz}, \mathrm{CDCl}_{3}\right) \delta 142.5,128.4,127.7,126.7,76.2,63.9,59.9,26.0,18.4,-4.3$, -4.9 .

HRMS (ESI): Calculated for $\mathrm{C}_{15} \mathrm{H}_{27} \mathrm{NO}_{2} \mathrm{Si}[\mathrm{M}+\mathrm{H}]^{+}:$: 282.1884, found: 282.1892.

TLC (dichloromethane : methanol = 9: 1) Rf: $0.38(\mathrm{KMnO} 4)$.

$[\boldsymbol{\alpha}]_{\mathbf{D}^{25}} \mathbf{2 5}+54.2\left(c 1.25, \mathrm{CHCl}_{3}\right)$. 


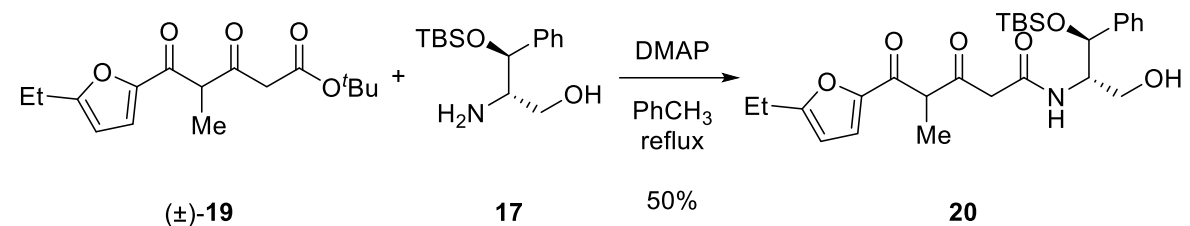

\section{$N$-((1S,2S)-1-((tert-Butyldimethylsilyl)oxy)-3-hydroxy-1-phenylpropan-2-yl)-5-(5- ethylfuran-2-yl)-4-methyl-3,5-dioxopentanamide (20):}

To a stirred solution of $( \pm)$-19 (355 mg, $1.21 \mathrm{mmol}, 1$ equiv) and (2S,3S)-2-amino-3((tert-butyldimethylsilyl)oxy)-3-phenylpropan-1-ol ${ }^{3}$ (17, $340 \mathrm{mg}, 1.21 \mathrm{mmol}, 1.00$ equiv) in toluene $(121 \mathrm{~mL})$ was added 4-dimethylaminopyridine (DMAP, $74.5 \mathrm{mg}, 0.603 \mathrm{mmol}, 0.50$ equiv) at $23{ }^{\circ} \mathrm{C}$, and the reaction flask was equipped with a reflux condenser. The resulting reaction mixture was heated to $115^{\circ} \mathrm{C}$. After $3 \mathrm{~h}$, the reaction mixture was cooled to $23^{\circ} \mathrm{C}$ and concentrated under reduced pressure. The resulting crude residue was purified by flash column chromatography (silica gel: diam. $4 \mathrm{~cm}$, ht. $11 \mathrm{~cm}$; eluent: ethyl acetate : hexanes $=1: 2$ to 3 : 1) to afford 20 (1:1 mixture of diastereomers, $302 \mathrm{mg}, 50 \%)$ as a yellow oil.

${ }^{1} \mathrm{H}$ NMR and ${ }^{13} \mathrm{C}$ NMR of 20 were taken with sample containing 1:1 mixture of inseparable diastereomers.

${ }^{1} \mathbf{H}$ NMR $\left(400.1 \mathrm{MHz}, \mathrm{CDCl}_{3}\right) \delta 7.39(\mathrm{~d}, J=8.1 \mathrm{~Hz}, 1 \mathrm{H}), 7.28-7.16(\mathrm{~m}, 13 \mathrm{H}), 6.20-6.16(\mathrm{~m}$, 2H), 4.95 (app-dd, $J=3.1,1.6 \mathrm{~Hz}, 2 \mathrm{H}$ ), 4.30 (app-dq, $J=12.0,7.0 \mathrm{~Hz}, 2 \mathrm{H}$ ), 3.99 (app-dtd, $J$ $=8.2,6.1,3.1 \mathrm{~Hz}, 2 \mathrm{H}), 3.68-3.56(\mathrm{~d}, J=7.0 \mathrm{~Hz}, 4 \mathrm{H}), 3.43-3.31(\mathrm{~m}, 4 \mathrm{H}), 2.78(\mathrm{br}-\mathrm{s}, 2 \mathrm{H})$, 2.74-2.64 (m, 4H), 1.36 (app-dd, $J=7.0,2.6 \mathrm{~Hz}, 6 \mathrm{H}), 1.25$ (app-td, $J=7.6,2.1 \mathrm{~Hz}, 6 \mathrm{H}), 0.90$ (s, 18H), $0.04(\mathrm{~s}, 6 \mathrm{H}),-0.19(\mathrm{~s}, 6 \mathrm{H})$.

${ }^{13}$ C NMR $\left(150.7 \mathrm{MHz}, \mathrm{CDCl}_{3}\right) \delta 202.5,202.2,185.0,184.8,166.1,165.9,164.6(2), 150.3$, 150.3, 141.8, 141.7, 128.3, 128.3, 127.7 (2), 126.3 (2), 121.1 (2), 108.4 (2), 73.0, 73.0, 63.2, 63.1, 58.5, 58.5, 56.5, 56.2, 48.4, 47.8, 26.0 (2), 22.0 (2), 18.3 (2), 13.2 (2), 11.8, 11.8, -4.5 (2), $-5.1(2)$.

HRMS (ESI): Calculated for $\mathrm{C}_{27} \mathrm{H}_{39} \mathrm{NO}_{6} \mathrm{Si}[\mathrm{M}+\mathrm{Na}]^{+}:$: 24.2439 , found: 524.2439.

TLC (ethyl acetate : hexanes = $2: 1) \mathrm{R} f: 0.34$ (CAM, UV).

\footnotetext{
${ }^{3}$ For a four-step synthesis of ent-17, see: Vatmurge, N. S.; Hazra, B. G.; Pore, V. S. Syntheses of 1,2-Amino Alcohols and Their Applications for Oxazaborolidine Catalyzed Enantioselective Reduction of Aromatic Ketones. Aust. J. Chem. 2007, 60, 196.
} 


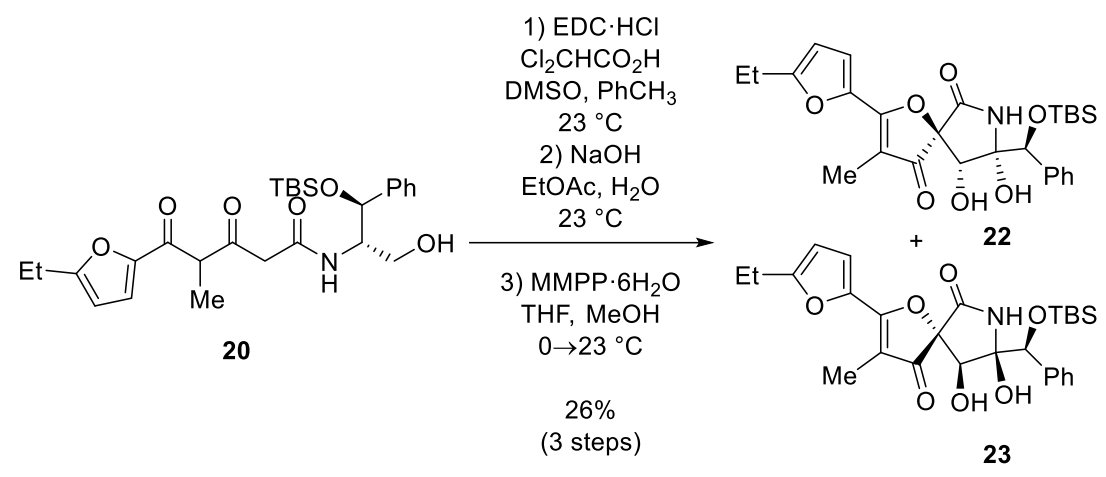

(5S,8S,9R)-8-((S)-((tert-Butyldimethylsilyl)oxy)(phenyl)methyl)-2-(5-ethylfuran-2-yl)-

8,9-dihydroxy-3-methyl-1-oxa-7-azaspiro[4.4]non-2-ene-4,6-dione $(22) \&(5 R, 8 R, 9 S)$-8((S)-((tert-butyldimethylsilyl)oxy)(phenyl)methyl)-2-(5-ethylfuran-2-yl)-8,9-dihydroxy-3methyl-1-oxa-7-azaspiro[4.4]non-2-ene-4,6-dione (23):

To a stirred solution of $\mathbf{2 0}$ (84.1 $\mathrm{mg}, 0.168 \mathrm{mmol}, 1$ equiv) in dimethylsulfoxide (1.7 $\mathrm{mL})$ and toluene $(1.7 \mathrm{~mL})$ were added 1-ethyl-3-(3-(dimethylamino)propyl) carbodiimide hydrochloride (EDC $\cdot \mathrm{HCl}, 164 \mathrm{mg}, 0.838 \mathrm{mmol}, 5.00$ equiv) and dichloroacetic acid $(27.9 \mu \mathrm{L}$, $0.335 \mathrm{mmol}, 2.00$ equiv) at $23{ }^{\circ} \mathrm{C}$. After $1 \mathrm{~h}$, saturated aqueous ammonium chloride solution $(6 \mathrm{~mL})$ was added to the reaction mixture, the resulting mixture was diluted with ethyl acetate $(6 \mathrm{~mL})$ and water $(6 \mathrm{~mL})$, and the layers were separated. The aqueous layer was extracted with ethyl acetate $(2 \times 6 \mathrm{~mL})$, and the organic layers were combined. Aqueous sodium hydroxide solution $(2.0 \mathrm{M}, 50 \mathrm{~mL})$ was added to the combined organic layers and stirred vigorously at $23{ }^{\circ} \mathrm{C}$. After $2 \mathrm{~h}$, the layers were separated. The aqueous layer was extracted with ethyl acetate $(2 \times 20 \mathrm{~mL})$, and the combined organic layers were washed with brine $(20 \mathrm{~mL})$, dried over anhydrous sodium sulfate, and concentrated under reduced pressure. The resulting crude residue of $\mathbf{2 6}$ was used in the next step without further purification.

To a stirred solution of $\mathbf{2 6}$ in a 1:1 mixture of tetrahydrofuran/methanol $(6.8 \mathrm{~mL})$ was added magnesium monoperoxyphthalate hexahydrate (MMPP. $6 \mathrm{H}_{2} \mathrm{O}, 80 \%, 114 \mathrm{mg}, 0.184$ mmol, 1.10 equiv) at $0{ }^{\circ} \mathrm{C}$. After $30 \mathrm{~min}$, the reaction mixture was allowed to warm to $23{ }^{\circ} \mathrm{C}$. After $1.5 \mathrm{~h}$, brine $(10 \mathrm{~mL})$ was added to the reaction mixture, the resulting mixture was diluted with ethyl acetate $(7 \mathrm{~mL})$, and the layers were separated. The aqueous layer was extracted with ethyl acetate $(2 \times 7 \mathrm{~mL})$, and the combined organic layers were dried over anhydrous sodium sulfate and concentrated under reduced pressure. The resulting crude residue was purified by flash column chromatography (silica gel: diam. $2.5 \mathrm{~cm}$, ht. $14 \mathrm{~cm}$; eluent: ethyl acetate : hexanes $=1: 4$ to $1: 2,2$ times $)$ to afford $\mathbf{2 2} \& \mathbf{2 3}(22.1 \mathrm{mg}, 26 \%)$ as a pale yellow oil. 
${ }^{1} \mathrm{H}$ NMR and ${ }^{13} \mathrm{C}$ NMR of $22 \& 23$ were taken with sample containing 1:1 mixture of inseparable diastereomers.

${ }^{1} \mathbf{H}$ NMR $\left(400.1 \mathrm{MHz}, \mathrm{CDCl}_{3}\right) \delta 7.44-7.29(\mathrm{~m}, 10 \mathrm{H}), 7.19$ (d, $\left.J=3.6 \mathrm{~Hz}, 1 \mathrm{H}\right), 7.11$ (d, $J=3.6$ $\mathrm{Hz}, 1 \mathrm{H}), 6.71$ (br-s, 1H), 6.26 (dt, $J=3.5,0.9 \mathrm{~Hz}, 1 \mathrm{H}), 6.22$ (dt, $J=3.7,0.9 \mathrm{~Hz}, 1 \mathrm{H}), 6.21$ (brs, 1H), 6.06 (br-s, 1H), 5.91 (br-s, 1H), $4.97(\mathrm{~d}, J=9.8 \mathrm{~Hz}, 1 \mathrm{H}), 4.83(\mathrm{~s}, 1 \mathrm{H}), 4.81(\mathrm{~s}, 1 \mathrm{H})$, $4.35(\mathrm{~d}, J=8.0 \mathrm{~Hz}, 1 \mathrm{H}), 2.93(\mathrm{~d}, J=10.2 \mathrm{~Hz}, 1 \mathrm{H}), 2.81-2.69(\mathrm{~m}, 5 \mathrm{H}), 1.98(\mathrm{~s}, 3 \mathrm{H}), 1.96(\mathrm{~s}$, $3 \mathrm{H}), 1.28(\mathrm{t}, J=7.6 \mathrm{~Hz}, 3 \mathrm{H}), 1.26(\mathrm{t}, J=7.6 \mathrm{~Hz}, 3 \mathrm{H}), 0.91(\mathrm{~s}, 9 \mathrm{H}), 0.87(\mathrm{~s}, 9 \mathrm{H}), 0.09(\mathrm{~s}, 3 \mathrm{H})$, $0.06(\mathrm{~s}, 3 \mathrm{H}),-0.11(\mathrm{~s}, 3 \mathrm{H}),-0.15(\mathrm{~s}, 3 \mathrm{H})$.

${ }^{13}$ C NMR $\left(150.7 \mathrm{MHz}, \mathrm{CDCl}_{3}\right) \delta$ 198.3, 198.1, 174.6, 174.3, 165.2, 165.0, 164.8, 164.7, 143.6, 143.4, 138.8, 138.5, 128.9, 128.7, 128.6, 128.6, 127.7 (2), 119.8 (2), 108.3, 108.3, 108.1 (2), $94.4,94.0,86.9,86.7,77.0,73.1,72.5,71.4,26.0,26.0,22.1,22.0,18.4(2), 11.9(2), 6.4,6.4$, $-4.4,-4.7,-4.8,-5.2$.

HRMS (ESI): Calculated for $\mathrm{C}_{27} \mathrm{H}_{35} \mathrm{NO}_{7} \mathrm{Si}[\mathrm{M}+\mathrm{Na}]^{+}:$536.2075, found: 536.2065.

TLC (ethyl acetate : hexanes = $1: 2$ ) Rf: 0.51 (CAM, UV). 


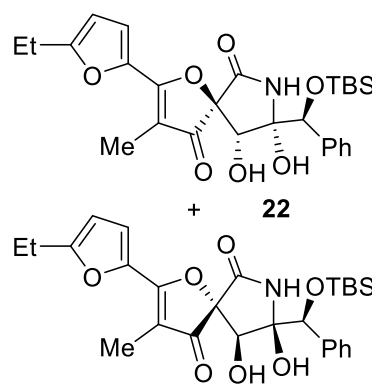

23

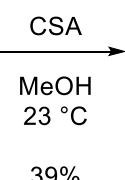

$39 \%$

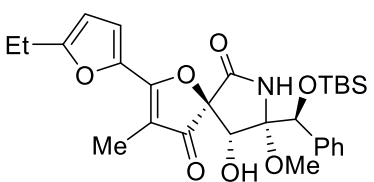

30

(5S,8S,9R)-8-((S)-((tert-Butyldimethylsilyl)oxy)(phenyl)methyl)-2-(5-ethylfuran-2-yl)-9hydroxy-8-methoxy-3-methyl-1-oxa-7-azaspiro[4-4]non-2-ene-4,6-dione (30):

To a stirred solution of $\mathbf{2 2} \& \mathbf{2 3}$ (10.2 $\mathrm{mg}, 0.020 \mathrm{mmol}, 1$ equiv) in methanol (3.0 mL) was added 10-camphorsulfonic acid (CSA, $60.0 \mathrm{mg}$ ) at $23{ }^{\circ} \mathrm{C}$. After $21 \mathrm{~h}$, saturated aqueous sodium bicarbonate solution $(2 \mathrm{~mL})$ was added to the reaction mixture, the resulting mixture was diluted with ethyl acetate $(10 \mathrm{~mL})$ and water $(5 \mathrm{~mL})$, and the layers were separated. The aqueous layer was extracted with ethyl acetate $(2 \times 10 \mathrm{~mL})$, and the combined organic layers were dried over anhydrous sodium sulfate and concentrated under reduced pressure. The resulting crude residue was purified by flash column chromatography (silica gel: diam. $3 \mathrm{~cm}$, ht. $12 \mathrm{~cm}$; eluent: ethyl acetate : hexanes $=1: 4$ to $1: 2)$ to afford $\mathbf{3 0}(4.1 \mathrm{mg}, 39 \%)$ as a pale yellow oil.

${ }^{1} \mathbf{H}$ NMR (400.1 MHz, $\left.\mathrm{CDCl}_{3}\right) \delta$ 7.34-7.29 (m, 5H), 7.07 (br-s, 1H), 6.91 (d, $\left.J=3.5 \mathrm{~Hz}, 1 \mathrm{H}\right)$, $6.15(\mathrm{dt}, J=3.5,0.9 \mathrm{~Hz}, 1 \mathrm{H}), 4.84(\mathrm{~s}, 1 \mathrm{H}), 4.36(\mathrm{~d}, J=10.8 \mathrm{~Hz}, 1 \mathrm{H}), 3.64(\mathrm{~s}, 3 \mathrm{H}), 3.52(\mathrm{~d}, J$ $=11.0 \mathrm{~Hz}, 1 \mathrm{H}), 2.70(\mathrm{q}, J=7.5 \mathrm{~Hz}, 2 \mathrm{H}), 1.93(\mathrm{~s}, 3 \mathrm{H}), 1.24(\mathrm{t}, J=7.6 \mathrm{~Hz}, 3 \mathrm{H}), 0.86(\mathrm{~s}, 9 \mathrm{H})$, $0.07(\mathrm{~s}, 3 \mathrm{H}),-0.26(\mathrm{~s}, 3 \mathrm{H})$.

${ }^{13}$ C NMR $\left(150.7 \mathrm{MHz}, \mathrm{CDCl}_{3}\right) \delta 196.2,172.2,167.8,163.6,143.9,138.8,128.9,128.6,127.9$, $117.8,108.2,107.7,91.8,88.8,78.7,73.5,53.5,26.0,22.0,18.2,12.0,6.3,-4.5,-4.9$.

HRMS (ESI): Calculated for $\mathrm{C}_{28} \mathrm{H}_{37} \mathrm{NO}_{7} \mathrm{Si}[\mathrm{M}+\mathrm{Na}]^{+}:$550.2232, found: 550.2235.

TLC (ethyl acetate : hexanes $=1: 2)$ Rf: $0.32($ CAM).

$[\boldsymbol{\alpha}]_{\mathbf{D}^{25}}$ + $57.0\left(\right.$ c $\left.0.47, \mathrm{CHCl}_{3}\right)$. 


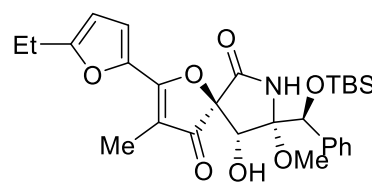

30

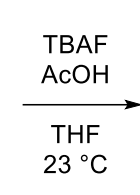

$50 \%$

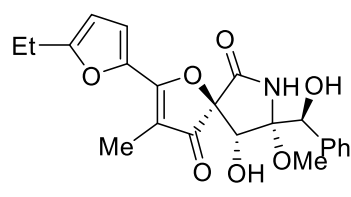

31

(5S,8R,9R)-2-(5-Ethylfuran-2-yl)-9-hydroxy-8-( $(S)$-hydroxy(phenyl)methyl)-8-methoxy3-methyl-1-oxa-7-azaspiro[4.4]non-2-ene-4,6-dione (31):

To a stirred solution of $\mathbf{3 0}$ (18.8 $\mathrm{mg}, 0.036 \mathrm{mmol}, 1$ equiv) and acetic acid (4.1 $\mu \mathrm{L}$, $0.071 \mathrm{mmol}, 2.00$ equiv) in tetrahydrofuran $(3.6 \mathrm{~mL})$, a solution of TBAF (1.0 M in THF, 143 $\mu \mathrm{L}, 0.143 \mathrm{mmol}, 4.00$ equiv) was added dropwise at $23^{\circ} \mathrm{C}$. After $20 \mathrm{~min}$, a solution of TBAF (1.0 $\mathrm{M}$ in THF, $143 \mu \mathrm{L}, 0.143 \mathrm{mmol}, 4.00$ equiv) was added dropwise at $23^{\circ} \mathrm{C}$. After $10 \mathrm{~min}$, brine $(7 \mathrm{~mL})$ was added to the reaction mixture, the resulting mixture was diluted with ethyl acetate $(5 \mathrm{~mL})$, and the layers were separated. The aqueous layer was extracted with ethyl acetate $(2 \times 5 \mathrm{~mL})$, and the combined organic layers were dried over anhydrous sodium sulfate and concentrated under reduced pressure. The resulting crude residue was purified by flash column chromatography (silica gel: diam. $2.5 \mathrm{~cm}$, ht. $8 \mathrm{~cm}$; eluent: ethyl acetate : hexanes = $1: 1$ to $3: 2)$ to afford $\mathbf{3 1}(7.3 \mathrm{mg}, 50 \%)$ as an orange oil.

${ }^{1} \mathbf{H}$ NMR (400.1 MHz, $\left.\mathrm{C}_{6} \mathrm{D}_{6}\right) \delta 8.19$ (br-s, 1H), 7.33-7.29 (m, 2H), 7.14-7.06 (m, 3H), 6.75 $(\mathrm{d}, J=3.5 \mathrm{~Hz}, 1 \mathrm{H}), 5.64(\mathrm{dt}, J=3.5,0.9 \mathrm{~Hz}, 1 \mathrm{H}), 5.18(\mathrm{~d}, J=9.5 \mathrm{~Hz}, 1 \mathrm{H}), 4.76(\mathrm{~s}, 1 \mathrm{H}), 3.66$ $(\mathrm{d}, J=10.7 \mathrm{~Hz}, 1 \mathrm{H}), 3.53(\mathrm{~s}, 3 \mathrm{H}), 3.35(\mathrm{br}-\mathrm{s}, 1 \mathrm{H}), 2.18(\mathrm{q}, J=7.5 \mathrm{~Hz}, 2 \mathrm{H}), 1.85(\mathrm{~s}, 3 \mathrm{H}), 0.84$ (t, $J=7.6 \mathrm{~Hz}, 3 \mathrm{H})$.

${ }^{13}$ C NMR $\left(100.6 \mathrm{MHz}, \mathrm{C}_{6} \mathrm{D}_{6}\right) \delta 196.6,172.3,169.5,163.2,144.5,139.1,128.6,128.5,128.1$, $117.8,109.0,107.8,93.1,89.5,74.9,73.8,52.6,21.9,11.9,6.6$.

HRMS (ESI): Calculated for $\mathrm{C}_{22} \mathrm{H}_{23} \mathrm{NO}_{7}[\mathrm{M}+\mathrm{Na}]^{+}$: 436.1367, found: 436.1366 .

TLC (ethyl acetate : hexanes $=2: 1) \mathrm{R} f: 0.21(\mathrm{CAM})$.

$[\alpha]_{\mathbf{D}^{25}}:+53.2\left(c 0.29, \mathrm{C}_{6} \mathrm{H}_{6}\right)$. 


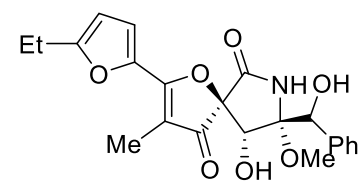

31

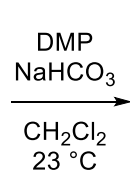

$63 \%$

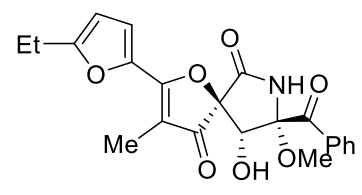

(-)-FD-838 (5)

\section{(-)-FD-838 (5):}

To a stirred solution of $\mathbf{3 1}$ ( $7.3 \mathrm{mg}, 0.018 \mathrm{mmol}, 1$ equiv) and sodium bicarbonate (14.8 mg, $0.177 \mathrm{mmol}, 10.0$ equiv) in dichloromethane $(5.3 \mathrm{~mL})$ was added Dess-Martin periodinane (DMP, $15.4 \mathrm{mg}, 0.035 \mathrm{mmol}, 2.00$ equiv) at $23{ }^{\circ} \mathrm{C}$. After $15 \mathrm{~min}$, saturated aqueous sodium bicarbonate solution $(5 \mathrm{~mL})$ was added to the reaction mixture, the resulting mixture was diluted with dichloromethane $(5 \mathrm{~mL})$, and the layers were separated. The aqueous layer was extracted with dichloromethane $(3 \times 5 \mathrm{~mL})$, and the combined organic layers were dried over anhydrous sodium sulfate and concentrated under reduced pressure. The resulting crude residue was purified by flash column chromatography (silica gel: diam. $3 \mathrm{~cm}$, ht. $12 \mathrm{~cm}$; eluent: ethyl acetate : hexanes $=1: 2)$ to afford $(-)$-FD-838 (5) $(4.6 \mathrm{mg}, 63 \%)$ as a pale yellow oil.

${ }^{1} \mathbf{H}$ NMR $\left(400.1 \mathrm{MHz}, \mathrm{CDCl}_{3}\right) \delta 8.32-8.28(\mathrm{~m}, 2 \mathrm{H}), 7.65-7.59(\mathrm{~m}, 1 \mathrm{H}), 7.50-7.44(\mathrm{~m}, 2 \mathrm{H})$, 7.36 (br-s, 1H), $7.02(\mathrm{~d}, J=3.6 \mathrm{~Hz}, 1 \mathrm{H}), 6.21(\mathrm{dt}, J=3.5,0.9 \mathrm{~Hz}, 1 \mathrm{H}), 4.67(\mathrm{~d}, J=12.5 \mathrm{~Hz}$, $1 \mathrm{H}), 4.09(\mathrm{~d}, J=12.5 \mathrm{~Hz}, 1 \mathrm{H}), 3.38(\mathrm{~s}, 3 \mathrm{H}), 2.73(\mathrm{q}, J=7.5 \mathrm{~Hz}, 2 \mathrm{H}), 1.99(\mathrm{~s}, 3 \mathrm{H}), 1.26(\mathrm{t}, J=$ $7.6 \mathrm{~Hz}, 3 \mathrm{H})$.

${ }^{13}$ C NMR $\left(150.7 \mathrm{MHz}, \mathrm{CDCl}_{3}\right) \delta 195.8,194.8,172.8,166.4,164.0,143.6,134.8,132.7,130.8$, $128.9,118.5,108.2,108.0,91.8,89.8,74.4,51.8,22.0,12.0,6.4$.

HRMS (ESI): Calculated for $\mathrm{C}_{22} \mathrm{H}_{21} \mathrm{NO}_{7}[\mathrm{M}+\mathrm{Na}]^{+}$: 434.1210 , found: 434.1211 .

TLC (ethyl acetate : hexanes = 1:1) Rf: 0.42 (CAM, UV).

$[\boldsymbol{\alpha}] \mathbf{D}^{\mathbf{2 6}}:-16.4\left(c 0.47, \mathrm{CHCl}_{3}\right)$.

$[\boldsymbol{\alpha}] \mathbf{D}^{22}:-14.2(c 0.49, \mathrm{EtOH})$. 


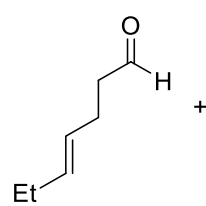

32

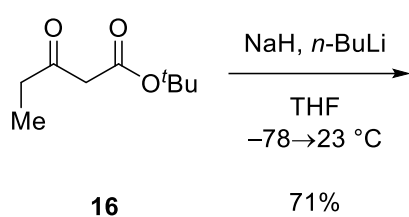

16

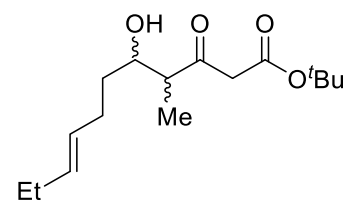

$( \pm)-54$

\section{tert-Butyl (E)-5-hydroxy-4-methyl-3-oxoundec-8-enoate (S4):}

To a suspension of sodium hydride ( $\mathrm{NaH}, 60 \%$ dispersion in mineral oil, $193 \mathrm{mg}, 4.82$ mmol, 1.10 equiv) in tetrahydrofuran $(11 \mathrm{~mL})$ at $0{ }^{\circ} \mathrm{C}$ was added tert-butyl 3-oxopentanoate (16, $779 \mu \mathrm{L}, 4.38 \mathrm{mmol}, 1$ equiv) dropwise under argon. After the reaction mixture was stirred at $0{ }^{\circ} \mathrm{C}$ for $30 \mathrm{~min}$, a solution of $n$-BuLi (2.50 M in $n$-hexane, $1.93 \mathrm{~mL}, 4.82 \mathrm{mmol}, 1.10$ equiv) was added dropwise to the reaction mixture. The reaction mixture was stirred at $0{ }^{\circ} \mathrm{C}$ for 30 $\min$. The resulting solution was cooled to $-78^{\circ} \mathrm{C}$. Subsequently, $(E)$-hept-4-enal ${ }^{4}(\mathbf{3 2}, 491 \mathrm{mg}$, $4.38 \mathrm{mmol}, 1.00$ equiv) in tetrahydrofuran $(11 \mathrm{~mL})$ was transferred by cannula to the reaction mixture. The reaction mixture was allowed to warm to $23{ }^{\circ} \mathrm{C}$. After $5 \mathrm{~h}$, saturated aqueous ammonium chloride solution $(20 \mathrm{~mL})$ was added to the reaction mixture, the resulting mixture was diluted with ethyl acetate $(40 \mathrm{~mL})$, and the layers were separated. The aqueous layer was extracted with ethyl acetate $(2 \times 40 \mathrm{~mL})$, and the combined organic layers were dried over anhydrous sodium sulfate and concentrated under reduced pressure. The resulting crude residue was purified by flash column chromatography (silica gel: diam. $5 \mathrm{~cm}$, ht. $10 \mathrm{~cm}$; eluent: ethyl acetate : hexanes $=1: 15$ to $1: 3)$ to afford $( \pm)$-S4 (1:1 mixture of diastereomers, $890 \mathrm{mg}, 71 \%)$ as a pale yellow oil.

${ }^{1} \mathrm{H}$ NMR and ${ }^{13} \mathrm{C}$ NMR of $\mathbf{S 4}$ were taken with sample containing 1:1 mixture of inseparable diastereomers.

${ }^{1} \mathbf{H}$ NMR $\left(400.1 \mathrm{MHz}, \mathrm{CDCl}_{3}\right) \delta 5.52-5.42(\mathrm{~m}, 2 \mathrm{H}), 5.41-5.31(\mathrm{~m}, 2 \mathrm{H}), 3.94(\mathrm{ddd}, J=9.0$, $4.2,3.1 \mathrm{~Hz}, 1 \mathrm{H}), 3.67$ (ddd, $J=9.2,7.2,3.1 \mathrm{~Hz}, 1 \mathrm{H}), 3.46-3.35(\mathrm{~m}, 4 \mathrm{H}), 2.77-2.64(\mathrm{~m}, 2 \mathrm{H})$, 2.44 (br-s, 2H), 2.20-2.01 (m, 4H), 2.01-1.90 (m, 4H), 1.60-1.33 (m, 4H), 1.43 (s, 18H), 1.10 (app-dd, $J=7.2,5.4 \mathrm{~Hz}, 6 \mathrm{H}$ ), 0.92 (app-td, $J=7.4,0.6 \mathrm{~Hz}, 6 \mathrm{H}$ ).

${ }^{13}$ C NMR $\left(150.7 \mathrm{MHz}, \mathrm{CDCl}_{3}\right) \delta 208.2,207.9,166.7,166.6,133.2,133.1,128.5,128.4,82.3$, $82.2,73.5,70.8,52.0,51.1,50.9,49.8,34.6,34.0,29.3,28.8,28.2$ (2), 25.7 (2), 14.1, 13.7, 9.8 (2).

HRMS (ESI): Calculated for $\mathrm{C}_{16} \mathrm{H}_{28} \mathrm{O}_{4}[\mathrm{M}+\mathrm{Na}]^{+}: 307.1880$, found: 307.1899 .

TLC (ethyl acetate : hexanes $=1: 3$ ) Rf: 0.50 (CAM, UV).

\footnotetext{
${ }^{4}$ Snyder, S. A.; Treitler, D. S.; Brucks, A. P.; Sattler, W. A General Strategy for the Stereocontrolled Preparation of Diverse 8- and 9-Membered Laurencia-Type Bromoethers. J. Am. Chem. Soc. 2011, 133, 15898.
} 
<smiles>CC/C=C/CC[C@@H](O)[C@H]([14CH3])C(=O)CC(=O)OCC</smiles>

$( \pm)-S 4$

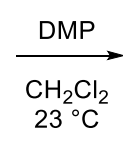

$82 \%$

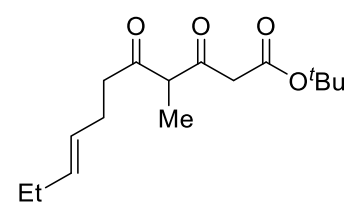

$( \pm)-33$

\section{tert-Butyl (E)-4-methyl-3,5-dioxoundec-8-enoate (33):}

To a stirred solution of $( \pm)$-S4 (756 mg, $2.66 \mathrm{mmol}, 1$ equiv) in dichloromethane (40 $\mathrm{mL}$ ) was added Dess-Martin periodinane (DMP, $1.69 \mathrm{~g}, 3.99 \mathrm{mmol}, 1.50$ equiv) at $23^{\circ} \mathrm{C}$. After $30 \mathrm{~min}$, a mixture of saturated aqueous sodium thiosulfate solution and saturated aqueous sodium bicarbonate solution $(1: 1,80 \mathrm{~mL})$ was added to the reaction mixture, and the layers were separated. The aqueous layer was extracted with dichloromethane $(2 \times 40 \mathrm{~mL})$, and the combined organic layers were dried over anhydrous sodium sulfate and concentrated under reduced pressure. The resulting crude residue was purified by flash column chromatography (silica gel: diam. $4 \mathrm{~cm}$, ht. $9 \mathrm{~cm}$; eluent: ethyl acetate : hexanes $=1: 10$ to $1: 7$ ) to afford $( \pm$ )$33(614 \mathrm{mg}, 82 \%)$ as a colorless oil. 33 was obtained as a mixture of keto/enol tautomers $($ keto/enol $=1 / 1)$.

${ }^{1} \mathbf{H}$ NMR $\left(400.1 \mathrm{MHz}, \mathrm{CDCl}_{3}\right) \delta 5.52-5.27(\mathrm{~m}, 4 \mathrm{H}), 3.81$ (q, $\left.J=7.1 \mathrm{~Hz}, 1 \mathrm{H}\right), 3.43-3.19(\mathrm{~m}$, $4 \mathrm{H}), 2.67-2.41(\mathrm{~m}, 4 \mathrm{H}), 2.32-2.17(\mathrm{~m}, 4 \mathrm{H}), 2.00-1.89(\mathrm{~m}, 4 \mathrm{H}), 1.79(\mathrm{~s}, 3 \mathrm{H}), 1.43(\mathrm{~s}, 9 \mathrm{H}), 1.42$ $(\mathrm{s}, 9 \mathrm{H}), 1.29(\mathrm{~d}, J=7.1 \mathrm{~Hz}, 3 \mathrm{H}), 0.95-0.87(\mathrm{~m}, 6 \mathrm{H})$.

${ }^{13}$ C NMR $\left(150.7 \mathrm{MHz}, \mathrm{CDCl}_{3}\right) \delta 206.6,200.3,194.6,184.2,167.2,166.3,133.6,133.4,127.4$, $127.1,105.3,82.4,82.0,60.5,49.3,44.1,41.6,36.6,28.2,28.1,28.1,26.6,25.7,25.7,13.9$, $13.9,12.7,12.5$.

HRMS (ESI): Calculated for $\mathrm{C}_{16} \mathrm{H}_{26} \mathrm{O}_{4}[\mathrm{M}+\mathrm{Na}]^{+}: 305.1723$, found: 305.1722 .

TLC (ethyl acetate : hexanes $=1: 4) \mathrm{R} f: 0.63$ (CAM, UV). 


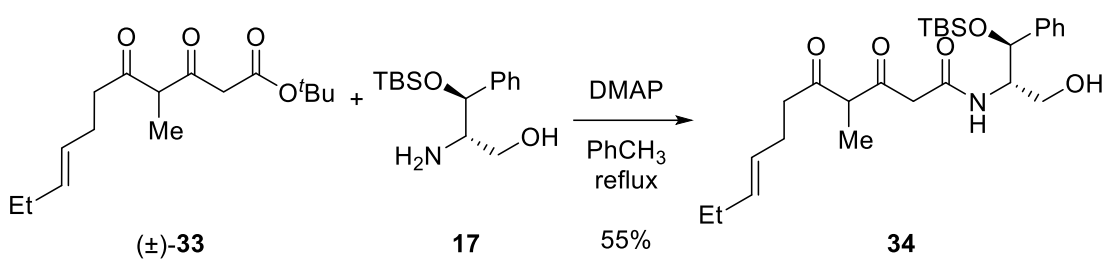

\section{(E)-N-((1S,2S)-1-((tert-Butyldimethylsilyl)oxy)-3-hydroxy-1-phenylpropan-2-yl)-4-}

\section{methyl-3,5-dioxoundec-8-enamide (34):}

To a stirred solution of $( \pm)$-33 (614 mg, $2.17 \mathrm{mmol}, 1$ equiv) and (2S,3S)-2-amino-3((tert-butyldimethylsilyl)oxy)-3-phenylpropan-1-ol ${ }^{3}$ (17, $612 \mathrm{mg}, 2.17 \mathrm{mmol}, 1.00$ equiv) in toluene $(217 \mathrm{~mL})$ was added 4-dimethylaminopyridine (DMAP, $134 \mathrm{mg}, 1.09 \mathrm{mmol}, 0.50$ equiv) at $23{ }^{\circ} \mathrm{C}$, and the reaction flask was equipped with a reflux condenser. The resulting reaction mixture was heated to $115^{\circ} \mathrm{C}$. After $3 \mathrm{~h}$, the reaction mixture was cooled to $23{ }^{\circ} \mathrm{C}$ and concentrated under reduced pressure. The resulting crude residue was purified by flash column chromatography (silica gel: diam. $4 \mathrm{~cm}$, ht. $10 \mathrm{~cm}$; eluent: ethyl acetate : hexanes $=1: 2$ to 3 : 1) to afford 34 (1:1 mixture of diastereomers, $586 \mathrm{mg}, 55 \%)$ as a yellow oil. 34 was obtained as a mixture of keto/enol tautomers (keto/enol $=1 / 1)$.

${ }^{1} \mathrm{H}$ NMR and ${ }^{13} \mathrm{C}$ NMR of $\mathbf{3 4}$ were taken with sample containing 1:1 mixture of inseparable diastereomers.

${ }^{1} \mathbf{H}$ NMR (400.1 MHz, $\left.\mathrm{CDCl}_{3}\right) \delta$ 7.35-7.15 (m, 10H), 7.10 (app-dd, $\left.J=8.0,3.7 \mathrm{~Hz}, 2 \mathrm{H}\right), 5.56$ $5.25(\mathrm{~m}, 4 \mathrm{H}), 4.99-4.93(\mathrm{~m}, 2 \mathrm{H}), 4.06-3.94(\mathrm{~m}, 2 \mathrm{H}), 3.74-3.66(\mathrm{~m}, 1 \mathrm{H}), 3.68-3.56(\mathrm{~m}, 4 \mathrm{H})$, 3.36-3.20 (m, 4H), 2.83 (br-s, 2H), 2.55-2.44 (m, 4H), 2.36-2.17 (m, 4H), 2.02-1.90 (m, 4H), 1.74 (s, 3H), 1.24 (app-dd, $J=7.1,3.9 \mathrm{~Hz}, 3 \mathrm{H}), 0.96-0.87$ (m, 24H), 0.07-0.00 (m, 6H), $0.16-0.22(\mathrm{~m}, 6 \mathrm{H})$.

${ }^{13}$ C NMR $\left(150.7 \mathrm{MHz}, \mathrm{CDCl}_{3}\right) \delta 207.2,206.9,203.0,202.7,195.7,184.1,166.8,165.9,165.8$, $142.0,141.9,141.9,133.6,133.5,133.5,128.2,128.1,128.1,127.5,127.5,127.4,127.2,126.9$, 126.9, $126.2(2), 126.0,105.7,72.5,72.5,72.1,62.5,62.4,62.4,60.6,60.1,58.4(2), 58.3,48.7$, $48.3,43.3,41.7,41.7,36.8,27.9,26.5,26.5,25.9(2), 25.9,25.6,25.6(2), 18.2(2), 18.2,13.8$ (3), $12.4(2), 12.3,-4.6(3),-5.2(2),-5.2$.

HRMS (ESI): Calculated for $\mathrm{C}_{27} \mathrm{H}_{43} \mathrm{NO}_{5} \mathrm{Si}[\mathrm{M}+\mathrm{Na}]^{+}:$: 512.2803, found: 512.2806.

TLC (ethyl acetate : hexanes = $2: 1) \mathrm{R} f: 0.45$ (CAM, UV). 


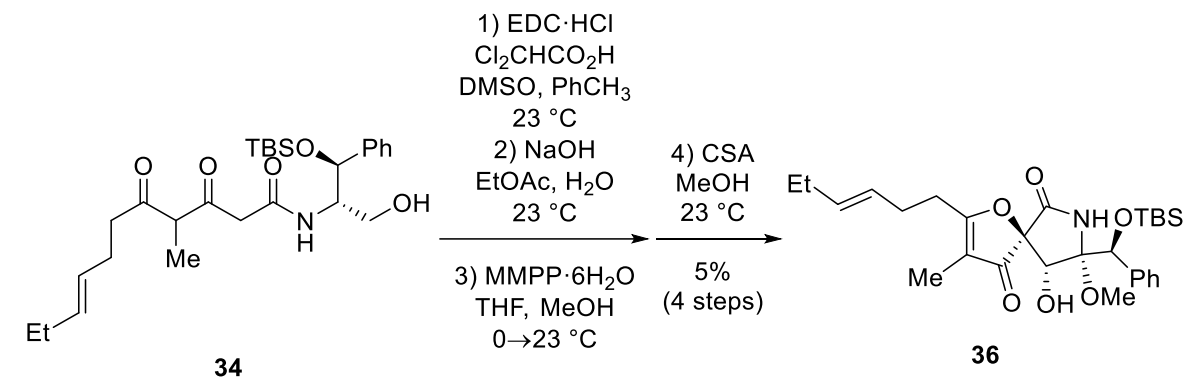

(5S,8S,9R)-8-((S)-((tert-Butyldimethylsilyl)oxy)(phenyl)methyl)-2-((E)-hex-3-en-1-yl)-9hydroxy-8-methoxy-3-methyl-1-oxa-7-azaspiro[4-4]non-2-ene-4,6-dione (36):

To a stirred solution of $\mathbf{3 4}$ (359 $\mathrm{mg}, 0.733 \mathrm{mmol}, 1$ equiv) in dimethylsulfoxide (7.3 $\mathrm{mL})$ and toluene $(7.3 \mathrm{~mL})$ were added 1-ethyl-3-(3-(dimethylamino)propyl) carbodiimide hydrochloride (EDC. $\mathrm{HCl}, 717 \mathrm{mg}, 3.67 \mathrm{mmol}, 5.00$ equiv) and dichloroacetic acid $(122 \mu \mathrm{L}$, $1.47 \mathrm{mmol}, 2.00$ equiv) at $23{ }^{\circ} \mathrm{C}$. After $1 \mathrm{~h}$, saturated aqueous ammonium chloride solution (25 $\mathrm{mL}$ ) was added to the reaction mixture, the resulting mixture was diluted with ethyl acetate ( 25 $\mathrm{mL})$ and water $(25 \mathrm{~mL})$, and the layers were separated. The aqueous layer was extracted with ethyl acetate $(2 \times 25 \mathrm{~mL})$, and the organic layers were combined. Aqueous sodium hydroxide solution $(2.0 \mathrm{M}, 140 \mathrm{~mL})$ was added to the combined organic layers and stirred vigorously at $23{ }^{\circ} \mathrm{C}$. After $2 \mathrm{~h}$, the layers were separated. The aqueous layer was extracted with ethyl acetate $(2 \times 30 \mathrm{~mL})$, and the combined organic layers were washed with brine $(30 \mathrm{~mL})$, dried over anhydrous sodium sulfate, and concentrated under reduced pressure. The resulting crude residue of $\mathbf{3 5}$ was used in the next step without further purification.

To a stirred solution of $\mathbf{3 5}$ in a 1:1 mixture of tetrahydrofuran/methanol (29.2 mL) was added magnesium monoperoxyphthalate hexahydrate (MMPP. $6 \mathrm{H}_{2} \mathrm{O}, 80 \%, 499 \mathrm{mg}, 0.806$ mmol, 1.10 equiv) at $0{ }^{\circ} \mathrm{C}$. After $30 \mathrm{~min}$, the reaction mixture was allowed to warm to $23{ }^{\circ} \mathrm{C}$. After $2 \mathrm{~h}$, brine $(30 \mathrm{~mL})$ was added to the reaction mixture, and the volatiles were removed under reduced pressure. The aqueous layer was extracted with ethyl acetate $(3 \times 15 \mathrm{~mL})$, and the combined organic layers were dried over anhydrous sodium sulfate and concentrated under reduced pressure. The resulting crude mixture was filtered through a silica pad (diam. $4 \mathrm{~cm}$, ht. $6 \mathrm{~cm}$ with $15 \%$ ethyl acetate in hexanes $600 \mathrm{~mL}$ ), and solvents were removed under reduced pressure.

To a stirred solution of the resulting mixture in methanol $(15.0 \mathrm{~mL})$ was added 10 camphorsulfonic acid (CSA, $300 \mathrm{mg}$ ) at $23{ }^{\circ} \mathrm{C}$. After $18 \mathrm{~h}$, saturated aqueous sodium bicarbonate solution $(5 \mathrm{~mL})$ and brine $(30 \mathrm{~mL})$ were added to the reaction mixture, and the volatile was removed under reduced pressure. The aqueous layer was extracted with ethyl acetate $(3 \times 20 \mathrm{~mL})$, and the combined organic layers were dried over anhydrous sodium sulfate and concentrated under reduced pressure. The resulting crude residue was purified by flash column chromatography (silica gel: diam. $4 \mathrm{~cm}$, ht. $9 \mathrm{~cm}$; eluent: ethyl acetate : hexanes $=1$ : 5 to $1: 2)$ to afford $\mathbf{3 6}(17.4 \mathrm{mg}, 5 \%)$ as a pale yellow oil. 
${ }^{1} \mathbf{H}$ NMR (400.1 MHz, $\left.\mathrm{CDCl}_{3}\right) \delta$ 7.36-7.27 (m, 5H), 6.81 (br-s, 1H), 5.54-5.44 (m, 1H), 5.39$5.29(\mathrm{~m}, 1 \mathrm{H}), 4.83(\mathrm{~s}, 1 \mathrm{H}), 4.22(\mathrm{~d}, J=10.5 \mathrm{~Hz}, 1 \mathrm{H}), 3.63(\mathrm{~s}, 3 \mathrm{H}), 3.49(\mathrm{~d}, J=11.0 \mathrm{~Hz}, 1 \mathrm{H})$, 2.56-2.48 (m, 2H), 2.30-2.22 (m, 2H), 2.00-1.90 (m, 2H), $1.58(\mathrm{~s}, 3 \mathrm{H}), 0.92$ (t, J= $7.4 \mathrm{~Hz}$, $3 \mathrm{H}), 0.86$ (s, 9H), 0.07 (s, 3H), -0.25 (s, 3H).

${ }^{13} \mathbf{C}$ NMR $\left(150.7 \mathrm{MHz}, \mathrm{CDCl}_{3}\right) \delta 197.5,190.0,167.5,138.8,134.3,128.9,128.6,127.8,126.5$, 111.0, 91.8, 88.7, 78.5, 73.2, 53.4, 29.4, 29.2, 26.0, 25.7, 18.2, 13.9, 5.8, -4.6, -5.0.

HRMS (ESI): Calculated for $\mathrm{C}_{28} \mathrm{H}_{41} \mathrm{NO}_{6} \mathrm{Si}[\mathrm{M}+\mathrm{Na}]^{+}:$538.2595, found: 538.2588.

TLC (ethyl acetate : hexanes = 1:2) Rf: 0.39 (CAM, UV).

$[\boldsymbol{\alpha}]_{\mathbf{D}^{25}} \mathbf{2}+32.2\left(c 0.33, \mathrm{CHCl}_{3}\right)$. 


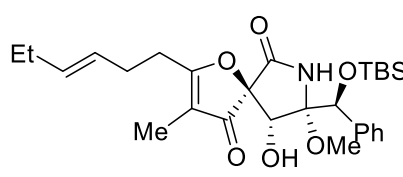

36

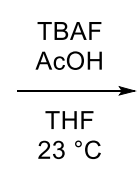

$57 \%$

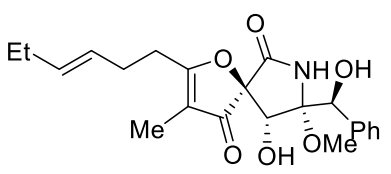

S5

$(5 S, 8 R, 9 R)-2-((E)-H e x-3-e n-1-y l)-9-h y d r o x y-8-((S)$-hydroxy(phenyl)methyl)-8-methoxy3-methyl-1-oxa-7-azaspiro[4.4]non-2-ene-4,6-dione (S5):

To a stirred solution of 36 (15.6 mg, $0.030 \mathrm{mmol}, 1$ equiv) and acetic acid ( $3.5 \mu \mathrm{L}$, $0.060 \mathrm{mmol}, 2.00$ equiv) in tetrahydrofuran $(3.0 \mathrm{~mL})$, a solution of TBAF (1.0 M in THF, 121 $\mu \mathrm{L}, 0.121 \mathrm{mmol}, 4.00$ equiv) was added dropwise at $23{ }^{\circ} \mathrm{C}$. After $20 \mathrm{~min}$, a solution of TBAF (1.0 $\mathrm{M}$ in THF, $90.7 \mu \mathrm{L}, 0.091 \mathrm{mmol}, 3.00$ equiv) was added dropwise at $23{ }^{\circ} \mathrm{C}$. After $10 \mathrm{~min}$, brine $(7 \mathrm{~mL})$ was added to the reaction mixture, the resulting mixture was diluted with ethyl acetate $(5 \mathrm{~mL})$, and the layers were separated. The aqueous layer was extracted with ethyl acetate $(2 \times 5 \mathrm{~mL})$, and the combined organic layers were dried over anhydrous sodium sulfate and concentrated under reduced pressure. The resulting crude residue was purified by flash column chromatography (silica gel: diam. $2.5 \mathrm{~cm}$, ht. $10 \mathrm{~cm}$; eluent: ethyl acetate : hexanes = $1: 1$ to $2: 1)$ to afford $\mathbf{S 5}(6.9 \mathrm{mg}, 57 \%)$ as a pale yellow oil.

${ }^{1} \mathbf{H}$ NMR $\left(400.1 \mathrm{MHz}, \mathrm{CD}_{2} \mathrm{Cl}_{2}\right) \delta$ 7.44-7.36 (m, 5H), 6.90 (br-s, 1H), 5.59-5.50 (m, 1H), 5.45$5.36(\mathrm{~m}, 1 \mathrm{H}), 4.88(\mathrm{~s}, 1 \mathrm{H}), 4.47(\mathrm{~d}, J=10.6 \mathrm{~Hz}, 1 \mathrm{H}), 3.56(\mathrm{~s}, 3 \mathrm{H}), 3.38(\mathrm{~d}, J=10.8 \mathrm{~Hz}, 1 \mathrm{H})$, 2.84 (br-s, 1H), 2.62-2.55 (m, 2H), 2.35-2.28 (m, 2H), 2.03-1.93 (m, 2H), 1.59 (s, 3H), 0.94 (t, $J=7.4 \mathrm{~Hz}, 3 \mathrm{H})$.

${ }^{13}$ C NMR $\left(150.7 \mathrm{MHz}, \mathrm{CD}_{2} \mathrm{Cl}_{2}\right) \delta 197.7,190.3,167.6,138.6,134.7,129.3,129.0,127.9,126.9$, $111.4,92.0,88.5,76.9,74.3,53.2,29.8,29.7,26.0,14.0,5.9$.

HRMS (ESI): Calculated for $\mathrm{C}_{22} \mathrm{H}_{27} \mathrm{NO}_{6}[\mathrm{M}+\mathrm{Na}]^{+}$: 424.1731, found: 424.1729 .

TLC (ethyl acetate : hexanes = $2: 1)$ Rf: 0.37 (CAM, UV).

$[\alpha]_{\mathbf{D}^{25}}$ +9.0 ( $\left.0.18, \mathrm{CH}_{2} \mathrm{Cl}_{2}\right)$. 


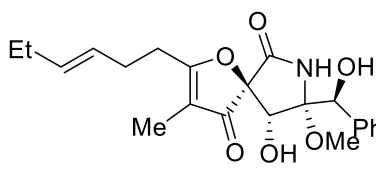

S5

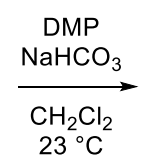

$75 \%$

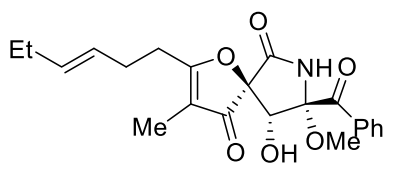

(-)-cephalimysin A (6)

\section{(-)-Cephalimysin A (6):}

To a stirred solution of $\mathbf{S 5}$ ( $3.5 \mathrm{mg}, 0.008 \mathrm{mmol}, 1$ equiv) and sodium bicarbonate (7.3 $\mathrm{mg}, 0.087 \mathrm{mmol}, 10.0$ equiv) in dichloromethane $(2.6 \mathrm{~mL})$ was added Dess-Martin periodinane (DMP, $5.7 \mathrm{mg}, 0.013 \mathrm{mmol}, 1.50$ equiv) at $23{ }^{\circ} \mathrm{C}$. After $20 \mathrm{~min}$, saturated aqueous sodium bicarbonate solution $(5 \mathrm{~mL})$ was added to the reaction mixture, the resulting mixture was diluted with dichloromethane $(3 \mathrm{~mL})$, and the layers were separated. The aqueous layer was extracted with dichloromethane $(2 \times 5 \mathrm{~mL})$, and the combined organic layers were dried over anhydrous sodium sulfate and concentrated under reduced pressure. The resulting crude residue was purified by flash column chromatography (silica gel: diam. $2 \mathrm{~cm}$, ht. $9 \mathrm{~cm}$; eluent: ethyl acetate : hexanes $=1: 2$ to $1: 1)$ to afford (-)-cephalimysin A (6) $(2.6 \mathrm{mg}, 75 \%)$ as a pale yellow oil.

${ }^{1} \mathbf{H}$ NMR $\left(400.1 \mathrm{MHz}, \mathrm{CDCl}_{3}\right) \delta 8.33-8.25(\mathrm{~m}, 2 \mathrm{H}), 7.65-7.59(\mathrm{~m}, 1 \mathrm{H}), 7.50-7.44(\mathrm{~m}, 2 \mathrm{H})$, 7.29 (br-s, 1H), 5.56-5.47 (m, 1H), 5.42-5.33 (m, 1H), 4.56 (d, $J=12.3 \mathrm{~Hz}, 1 \mathrm{H}), 4.03$ (d, $J=$ $12.5 \mathrm{~Hz}, 1 \mathrm{H}), 3.36(\mathrm{~s}, 3 \mathrm{H}), 2.69-2.53(\mathrm{~m}, 2 \mathrm{H}), 2.39-2.29(\mathrm{~m}, 2 \mathrm{H}), 2.04-1.90(\mathrm{~m}, 2 \mathrm{H}), 1.65$ (s, $3 \mathrm{H}), 0.90(\mathrm{t}, J=7.5 \mathrm{~Hz}, 3 \mathrm{H})$.

${ }^{13}$ C NMR $\left(150.7 \mathrm{MHz}, \mathrm{CDCl}_{3}\right) \delta 197.3,194.8,190.8,166.0,134.8,134.6,132.7,130.8,128.9$, $126.3,111.3,91.8,89.8,74.3,51.8,29.6,29.3,25.7,13.8,5.9$.

HRMS (ESI): Calculated for $\mathrm{C}_{22} \mathrm{H}_{25} \mathrm{NO}_{6}[\mathrm{M}+\mathrm{Na}]^{+}:$422.1574, found: 422.1574 .

TLC (ethyl acetate : hexanes $=1: 1) \mathrm{R} f: 0.50$ (CAM, UV).

$[\boldsymbol{\alpha}]_{\mathrm{D}}{ }^{25}:-32.6(c 0.53, \mathrm{EtOH})$. 
Table S1. Comparison of ${ }^{1} \mathrm{H}$ NMR Spectral Data of Our Synthetic (-)-FD-838 (5) with Isolation Literature

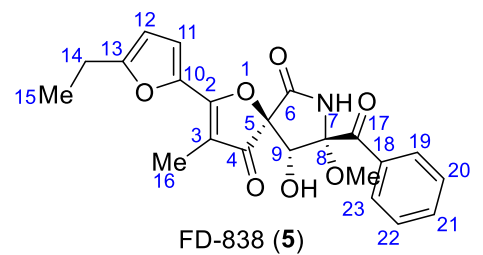

\begin{tabular}{|c|c|c|}
\hline Assignment & $\begin{array}{l}\text { Yamada's Report }^{5} \\
{ }^{1} \mathrm{H} \text { NMR, } \mathrm{CDCl}_{3}\end{array}$ & $\begin{array}{l}\text { This Work }^{6} \\
{ }^{1} \mathrm{H} \mathrm{NMR}, 400.1 \mathrm{MHz}, \mathrm{CDCl}_{3}\end{array}$ \\
\hline N7 & 7.44 (br-s, 1H) & 7.36 (br-s, 1H) \\
\hline C9 & $4.69(\mathrm{~d}, J=12.7 \mathrm{~Hz}, 1 \mathrm{H})$ & $4.67(\mathrm{~d}, J=12.5 \mathrm{~Hz}, 1 \mathrm{H})$ \\
\hline C11 & $7.04(\mathrm{~d}, J=3.4 \mathrm{~Hz}, 1 \mathrm{H})$ & $7.02(\mathrm{~d}, J=3.6 \mathrm{~Hz}, 1 \mathrm{H})$ \\
\hline C12 & $6.23(\mathrm{~d}, J=3.4 \mathrm{~Hz}, 1 \mathrm{H})$ & $6.21(\mathrm{dt}, J=3.5,0.9 \mathrm{~Hz}, 1 \mathrm{H})$ \\
\hline C14 & $2.75(\mathrm{q}, J=7.6 \mathrm{~Hz}, 2 \mathrm{H})$ & $2.73(\mathrm{q}, J=7.5 \mathrm{~Hz}, 2 \mathrm{H})$ \\
\hline C15 & $1.28(\mathrm{t}, J=7.6 \mathrm{~Hz}, 3 \mathrm{H})$ & $1.26(\mathrm{t}, J=7.6 \mathrm{~Hz}, 3 \mathrm{H})$ \\
\hline C16 & $2.01(\mathrm{~s}, 3 \mathrm{H})$ & $1.99(\mathrm{~s}, 3 \mathrm{H})$ \\
\hline C19(C23) & $8.32(\mathrm{~d}, J=8.5 \mathrm{~Hz}, 2 \mathrm{H})$ & $8.32-8.28(\mathrm{~m}, 2 \mathrm{H})$ \\
\hline C20(22) & $7.49(\mathrm{t}, J=7.6 \mathrm{~Hz}, 2 \mathrm{H})$ & $7.50-7.44(\mathrm{~m}, 2 \mathrm{H})$ \\
\hline C21 & $7.64(\mathrm{t}, J=7.6 \mathrm{~Hz}, 1 \mathrm{H})$ & 7.65-7.59 (m, 1H) \\
\hline 8-OMe & $3.40(\mathrm{~s}, 3 \mathrm{H})$ & $3.38(\mathrm{~s}, 3 \mathrm{H})$ \\
\hline $9-\mathrm{OH}$ & $4.13(\mathrm{~d}, J=12.7 \mathrm{~Hz}, 1 \mathrm{H})$ & $4.09(\mathrm{~d}, J=12.5 \mathrm{~Hz}, 1 \mathrm{H})$ \\
\hline
\end{tabular}

${ }^{5}$ Yamada, T.; Kitada, H.; Kajimoto, T.; Numata, A.; Tanaka, R. The Relationship between the CD Cotton Effect and the Absolute Configuration of FD-838 and Its Seven Stereoisomers. J. Org. Chem. 2010, 75, 4146.

${ }^{6}$ The chemical shifts were recorded with respect to the deuterated solvent shift ( $\mathrm{CDCl}_{3}, \delta 7.24$ for the hydrogen). 
Table S2. Comparison of ${ }^{13} \mathrm{C}$ NMR Spectral Data of Our Synthetic (-)-FD-838 (5) with Isolation Literature

\begin{tabular}{|c|c|c|}
\hline Assignment & $\begin{array}{l}\text { Yamada's Report }{ }^{5} \\
{ }^{13} \mathrm{C} \mathrm{NMR}_{,} \mathrm{CDCl}_{3}\end{array}$ & $\begin{array}{l}\text { This Work }^{7} \\
{ }^{13} \mathrm{C} \mathrm{NMR}, 150.7 \mathrm{MHz}, \mathrm{CDCl}_{3}\end{array}$ \\
\hline C2 & 172.5 & 172.8 \\
\hline C3 & 107.8 & $108.2^{8}$ \\
\hline C4 & 195.6 & 195.8 \\
\hline C5 & 89.6 & $91.8^{9}$ \\
\hline C6 & 166.2 & 166.4 \\
\hline C8 & 91.5 & $89.8^{9}$ \\
\hline C9 & 74.2 & 74.4 \\
\hline C10 & 143.3 & 143.6 \\
\hline C11 & 118.3 & 118.5 \\
\hline C12 & 107.9 & $108.0^{8}$ \\
\hline C13 & 163.7 & 164.0 \\
\hline C14 & 21.8 & 22.0 \\
\hline C15 & 11.8 & 12.0 \\
\hline C16 & 6.2 & 6.4 \\
\hline C17 & 194.6 & 194.8 \\
\hline C18 & 132.4 & 132.7 \\
\hline C19(C23) & 130.6 & 130.8 \\
\hline C20(C22) & 128.7 & 128.9 \\
\hline C21 & 134.5 & 134.8 \\
\hline 8-OMe & 51.6 & 51.8 \\
\hline
\end{tabular}

\footnotetext{
7 The chemical shifts were recorded with respect to the deuterated solvent shift ( $\mathrm{CDCl}_{3}, \delta 77.23$ for the carbon).

${ }^{8}{ }^{13} \mathrm{C}$ NMR chemical shifts have been reassigned based on HMBC data. Key HMBC correlations: H14-C12, H16-C3.

$9{ }^{13} \mathrm{C}$ NMR chemical shifts have been reassigned based on HMBC data. Key HMBC correlations: 8-OMe-C8.
} 
Table S3. Comparison of ${ }^{1} \mathrm{H}$ NMR Spectral Data of Our Synthetic (-)-cephalimysin A (6) with Isolation Literature

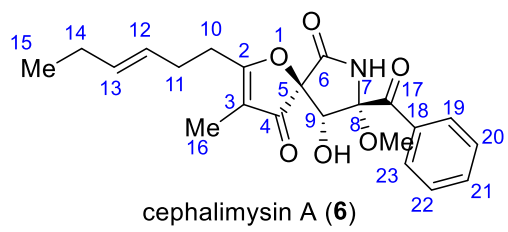

\begin{tabular}{|c|c|c|}
\hline Assignment & $\begin{array}{l}\text { Yamada's Report }{ }^{10} \\
{ }^{1} \mathrm{H} \mathrm{NMR}, \mathrm{CDCl}_{3}\end{array}$ & $\begin{array}{l}\text { This Work }^{6} \\
{ }^{1} \mathrm{H} \mathrm{NMR}, 400.1 \mathrm{MHz}, \mathrm{CDCl}_{3}\end{array}$ \\
\hline N7 & $7.41(\mathrm{br}-\mathrm{s}, 1 \mathrm{H})$ & 7.29 (br-s, 1H) \\
\hline C9 & $4.59(\mathrm{~d}, J=12.5 \mathrm{~Hz}, 1 \mathrm{H})$ & $4.56(\mathrm{~d}, J=12.3 \mathrm{~Hz}, 1 \mathrm{H})$ \\
\hline C10 & $2.62(\mathrm{~m}, 2 \mathrm{H})$ & $2.69-2.53(\mathrm{~m}, 2 \mathrm{H})$ \\
\hline C11 & $2.35(\mathrm{~m}, 2 \mathrm{H})$ & $2.39-2.29(\mathrm{~m}, 2 \mathrm{H})$ \\
\hline $\mathrm{C} 12$ & $5.40(\mathrm{dt}, J=15.1,6.9 \mathrm{~Hz}, 1 \mathrm{H})$ & $5.42-5.33(\mathrm{~m}, 2 \mathrm{H})$ \\
\hline C13 & $5.54(\mathrm{dt}, J=15.1,6.2 \mathrm{~Hz}, 1 \mathrm{H})$ & $5.56-5.47(\mathrm{~m}, 2 \mathrm{H})$ \\
\hline C14 & $1.97(\mathrm{~m}, 2 \mathrm{H})$ & $2.04-1.90(m, 2 H)$ \\
\hline C15 & $0.93(\mathrm{t}, J=7.1 \mathrm{~Hz}, 3 \mathrm{H})$ & $0.90(\mathrm{t}, J=7.5 \mathrm{~Hz}, 3 \mathrm{H})$ \\
\hline C16 & $1.68(\mathrm{~s}, 3 \mathrm{H})$ & $1.65(\mathrm{~s}, 3 \mathrm{H})$ \\
\hline C19(C23) & $8.31(\mathrm{~d}, J=7.9 \mathrm{~Hz}, 2 \mathrm{H})$ & $8.33-8.25(\mathrm{~m}, 2 \mathrm{H})$ \\
\hline C20(C22) & $7.48(\mathrm{t}, J=7.9 \mathrm{~Hz}, 2 \mathrm{H})$ & $7.50-7.44(\mathrm{~m}, 2 \mathrm{H})$ \\
\hline $\mathrm{C} 21$ & $7.64(\mathrm{t}, J=7.9 \mathrm{~Hz}, 1 \mathrm{H})$ & $7.65-7.59(\mathrm{~m}, 1 \mathrm{H})$ \\
\hline 8-OMe & $3.40(\mathrm{~s}, 3 \mathrm{H})$ & $3.36(\mathrm{~s}, 3 \mathrm{H})$ \\
\hline $9-\mathrm{OH}$ & $4.06(\mathrm{~d}, J=12.5 \mathrm{~Hz}, 1 \mathrm{H})$ & $4.03(\mathrm{~d}, J=12.5 \mathrm{~Hz}, 1 \mathrm{H})$ \\
\hline
\end{tabular}

10 Yamada, T.; Imai, E.; Nakatuji, K.; Numata, A.; Tanaka, R. Cephalimysin A, a potent cytotoxic metabolite from an Aspergillus species separated from a marine fish. Tetrahedron Lett. 2007, 48, 6294. 
Table S4. Comparison of ${ }^{13}$ C NMR Spectral Data of Our Synthetic (-)-cephalimysin A (6) with Isolation Literature

\begin{tabular}{|c|c|c|}
\hline Assignment & $\begin{array}{l}\text { Yamada's Report }{ }^{10} \\
{ }^{13} \mathrm{C} \mathrm{NMR}_{,} \mathrm{CDCl}_{3}\end{array}$ & $\begin{array}{l}\text { This Work }^{7} \\
{ }^{13} \mathrm{C} \mathrm{NMR}, 150.7 \mathrm{MHz}, \mathrm{CDCl}_{3}\end{array}$ \\
\hline C2 & 190.54 & 190.76 \\
\hline C3 & 111.06 & 111.29 \\
\hline C4 & 197.11 & 197.32 \\
\hline C5 & 91.52 & 91.75 \\
\hline C6 & 165.75 & 165.97 \\
\hline C8 & 89.62 & 89.84 \\
\hline C9 & 74.06 & 74.30 \\
\hline C10 & 29.36 & 29.60 \\
\hline C11 & 29.04 & 29.28 \\
\hline C12 & 126.04 & 126.28 \\
\hline C13 & 134.36 & 134.60 \\
\hline C14 & 25.44 & 25.68 \\
\hline C15 & 13.61 & 13.84 \\
\hline C16 & 5.67 & 5.90 \\
\hline C17 & 194.58 & 194.80 \\
\hline C18 & 132.41 & 132.65 \\
\hline C19(C23) & 130.55 & 130.79 \\
\hline C20(C22) & 128.67 & 128.90 \\
\hline C21 & 134.52 & 134.76 \\
\hline 8-OMe & 51.60 & 51.83 \\
\hline
\end{tabular}


HPLC Traces of Synthetic (-)-FD-838, (+)-FD-838, and Their Mixture

Figure S1. HPLC trace of a mixture of (-)-FD-838 and (+)-FD-838 (Both enantiomers were synthesized separately and then mixed, CHIRALPAK IB-3, hexanes : $i$-PrOH $=80: 20,0.5$ $\mathrm{mL} / \mathrm{min}$ ).

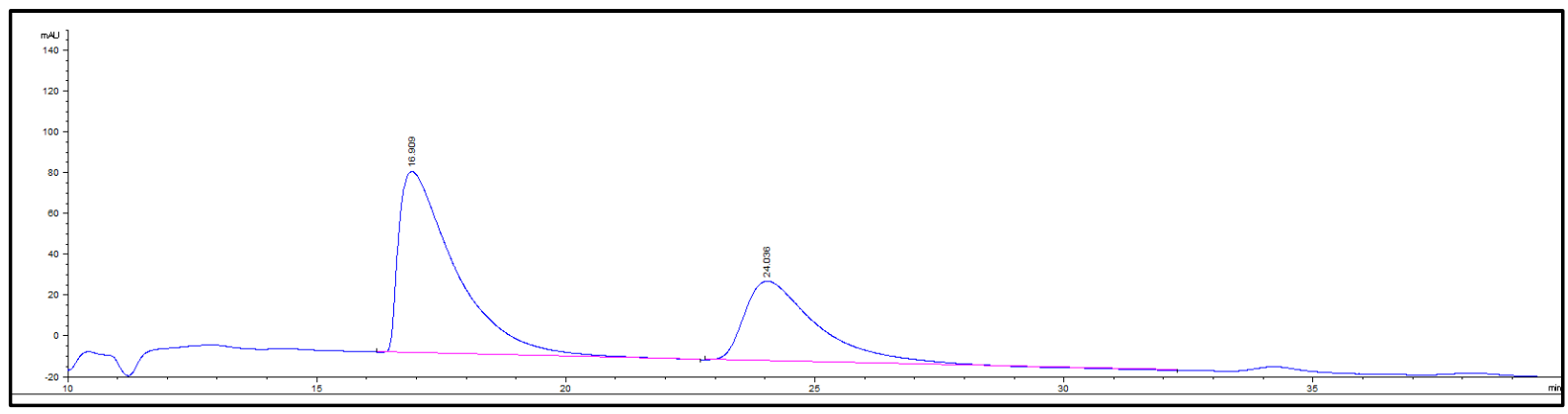

Figure S2. HPLC trace of synthetic (-)-FD-838 (CHIRALPAK IB-3, hexanes : $i$-PrOH $=80$ : $20,0.5 \mathrm{~mL} / \mathrm{min})$.

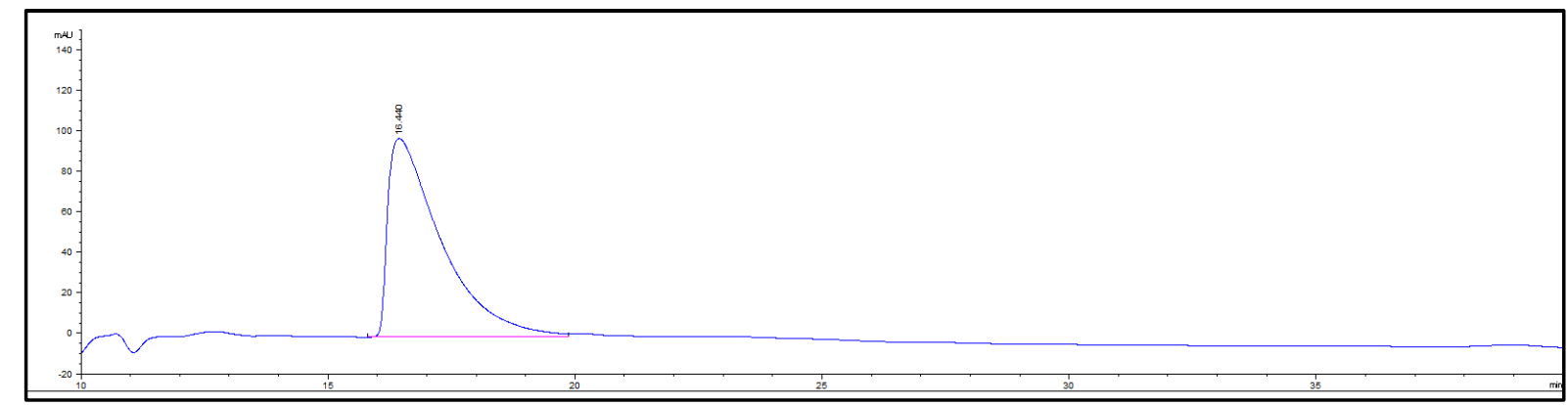

Figure S3. HPLC trace of synthetic (+)-FD-838 (CHIRALPAK IB-3, hexanes : $i$-PrOH $=80$ : $20,0.5 \mathrm{~mL} / \mathrm{min})$.

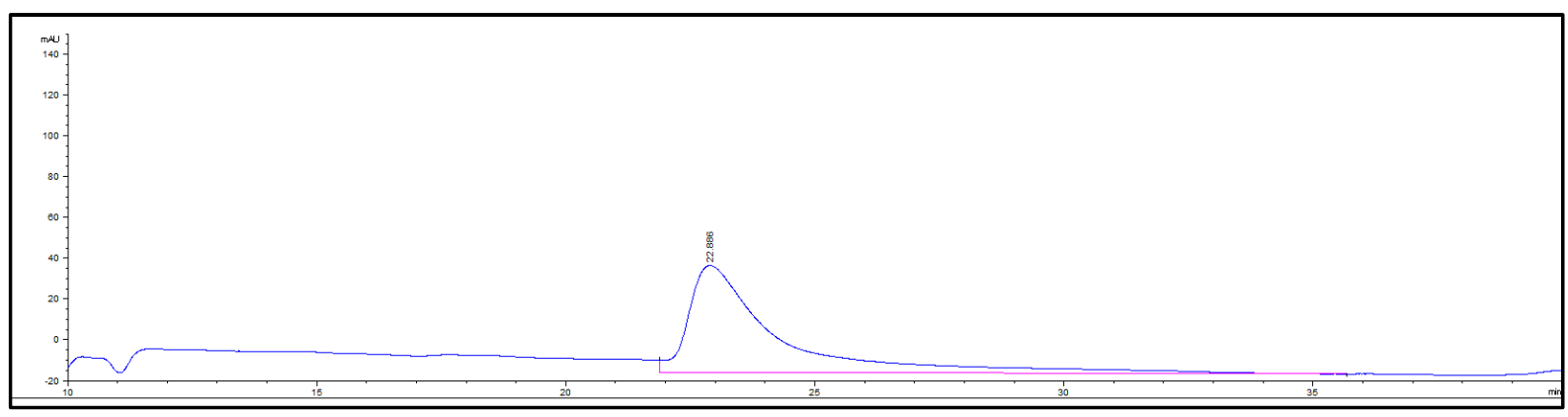


Confirmation of the Absolute Stereochemistry of Our Synthetic (-)-FD-838

Table S5. Comparison of specific rotation data of FD-838

\begin{tabular}{|c|c|}
\hline Source of sample & Specific rotation \\
\hline Mizoue's isolation report $^{11}$ & {$[\alpha]_{\mathrm{D}}^{26}: 0(c 0.1, \mathrm{MeOH})$} \\
\hline Hayashi's synthesis $^{12}$ & Synthetic sample $[\alpha]_{\mathrm{D}}^{26}:-41.9\left(c 0.4, \mathrm{CHCl}_{3}\right)$ \\
\hline Yamada's isolation report $^{5}$ & Natural sample $[\alpha]_{\mathrm{D}}^{26}:-32.4\left(c 0.7, \mathrm{CHCl}_{3}\right)$ \\
\hline This report & {$[\alpha]_{\mathrm{D}}^{22}:+40.5(c 0.10, \mathrm{EtOH})$} \\
\hline & {$[\alpha]_{\mathrm{D}}^{22}:-16.4(c 0.47, \mathrm{CHCl} 3)$} \\
& {$[\alpha]_{\mathrm{D}}{ }^{26}:-14.2(c 0.49, \mathrm{EtOH})$} \\
\hline
\end{tabular}

Figure S4. Comparison of circular dichroism (CD) spectra of FD-838

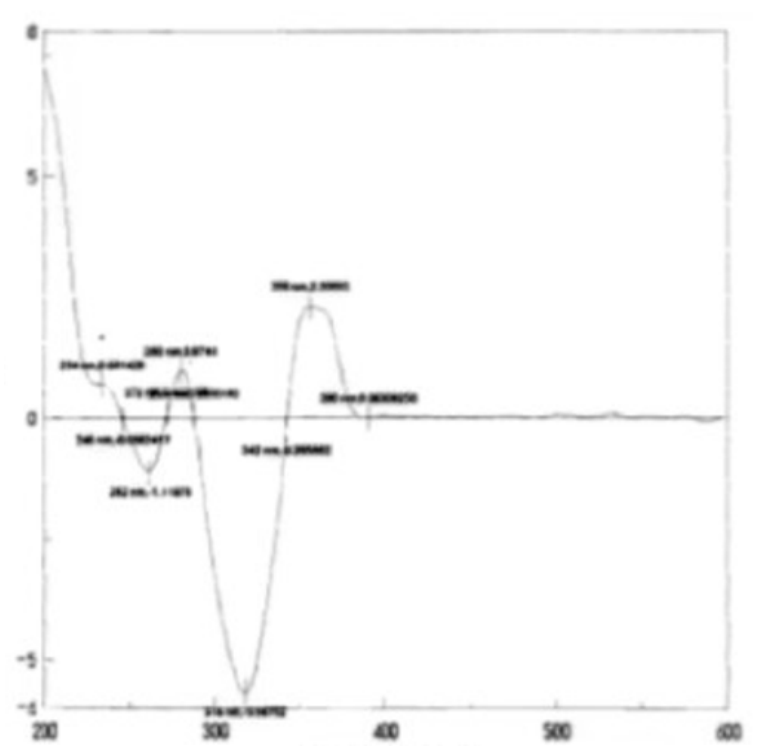

CD spectrum of natural FD- $838^{12}$

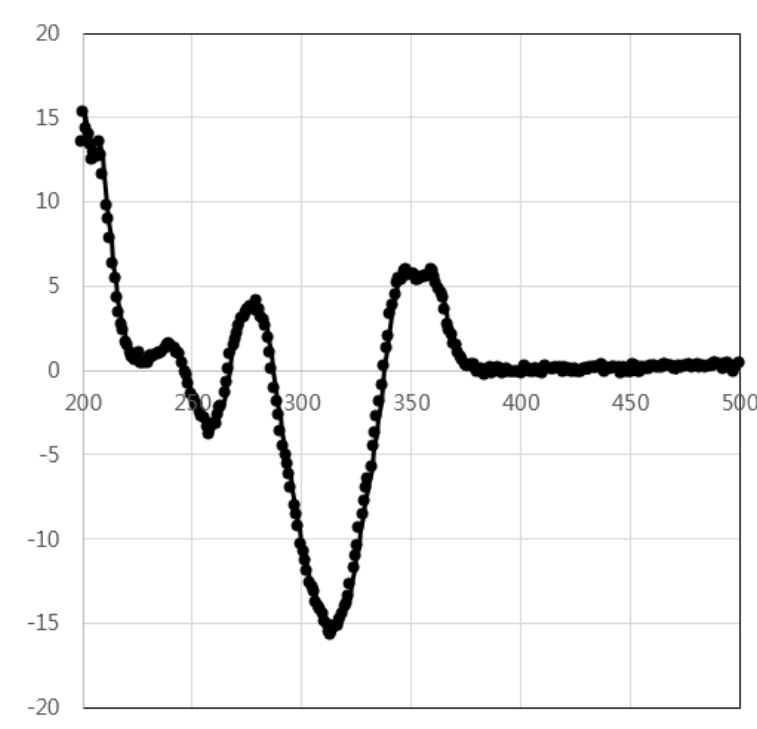

CD spectrum of our synthetic FD-838

\footnotetext{
11 Mizoue, K.; Okazaki, T.; Hanada, K.; Amamoto, T.; Yamagishi, M.; Omura, S. Physiologically active substance FD-838 and process for preparing the same. Eur. Pat. Appl. EP 216607 A2, 1987.

${ }^{12}$ Hayashi, Y.; Sankar, K.; Ishikawa, H.; Nozawa, Y.; Mizoue, K.; Kakeya, H. Total synthesis and determination of the absolute configuration of FD-838, a naturally occurring azaspirobicyclic product. Bioorg. Med. Chem. Lett. 2009, 19, 3863.
} 
Comparison of the CD Spectra of Our Synthetic (-)-Cephalimysin A and the Natural Sample

Figure S5. CD spectrum of the natural cephalimysin A (ethanol solution) isolated by the Yamada group. ${ }^{10,13}$

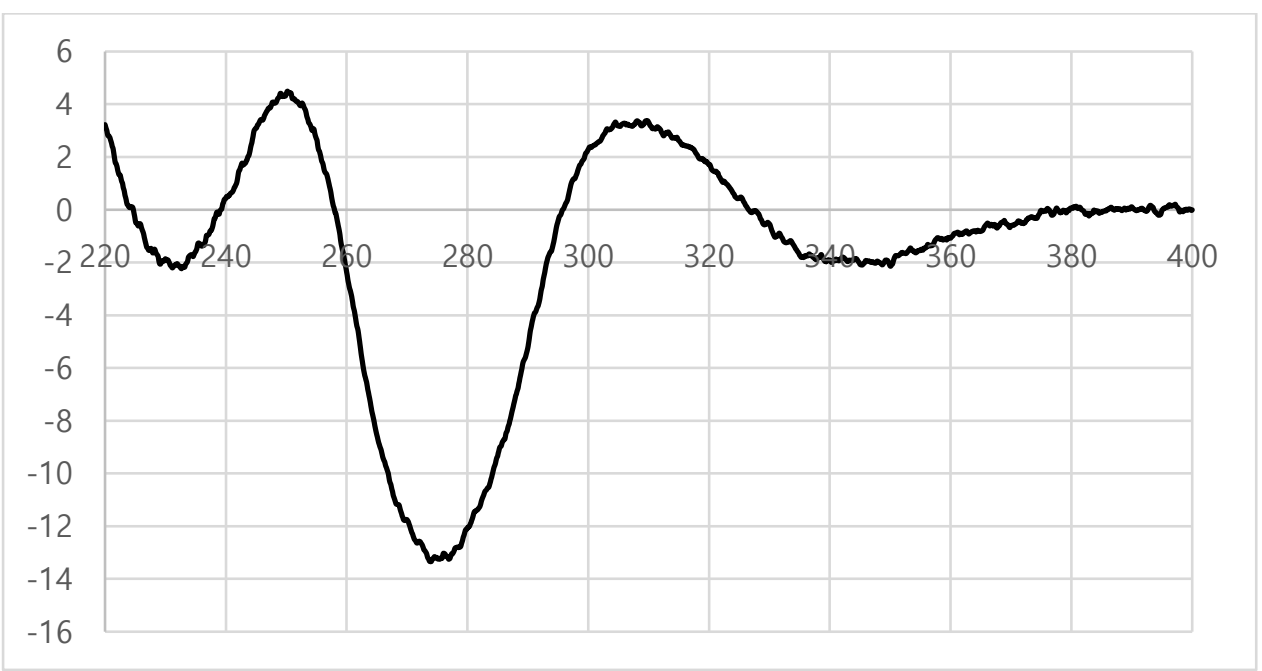

Figure S6. CD spectrum of our synthetic (-)-cephalimysin A (6, ethanol solution).

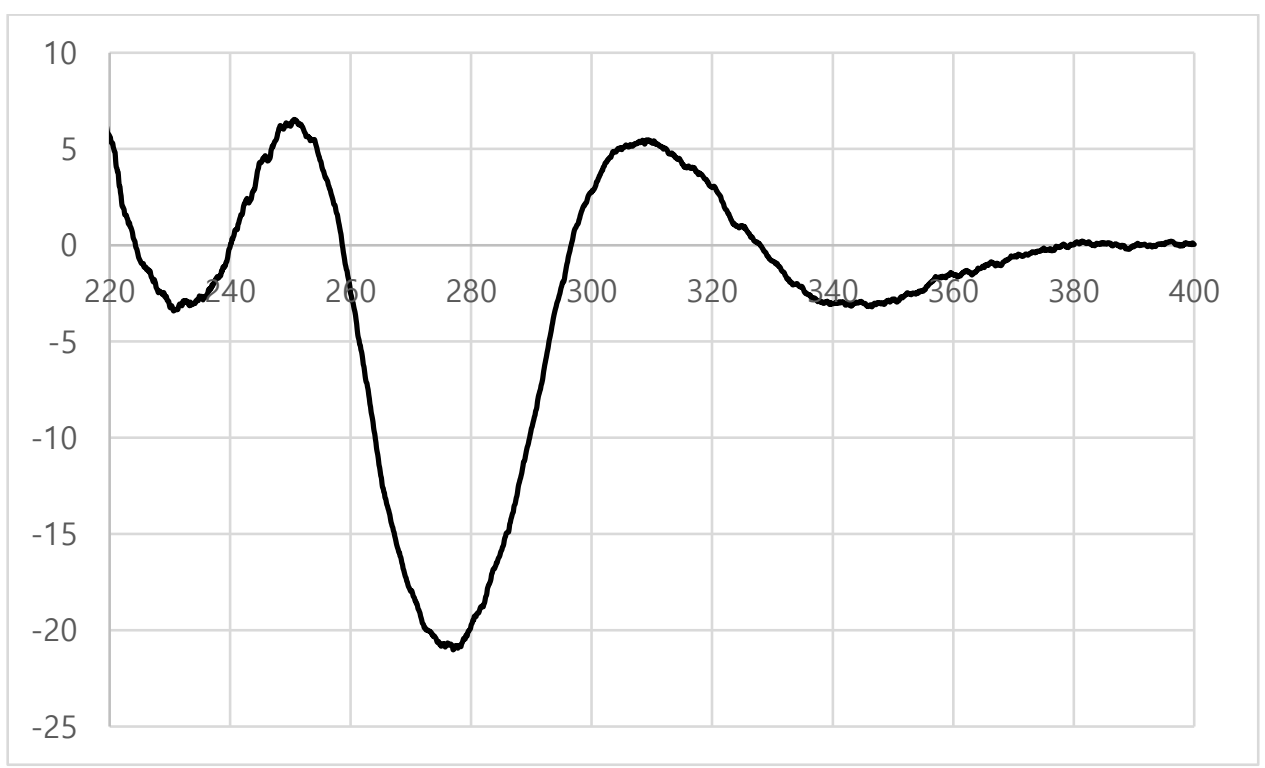

13 We thank Dr. Takeshi Yamada for kindly providing us with the CD spectral data of the natural sample of cephalimysin A. 


\begin{tabular}{|lll|}
\hline \multicolumn{1}{|c|}{ Parameter } & \multicolumn{1}{c|}{ Value } \\
1 & Origin & Bruker BioSpin GmbH \\
2 & Spectrometer & spect \\
3 & Solvent & $\mathrm{CDCl} 3$ \\
4 & Temperature & 298.0 \\
5 & Pulse Sequence & zg30 \\
6 & Experiment & 10 \\
7 & Probe & $5 \mathrm{~mm} \mathrm{PABBO} \mathrm{BB/} \mathrm{19F-1H/} \mathrm{D} \mathrm{Z-GRD} \mathrm{Z108618/} \mathrm{0389}$ \\
8 & Number of Scans & 31 \\
9 & Receiver Gain & 113 \\
10 Relaxation Delay & 0.2000 \\
11 Pulse Width & 15.0000 \\
12 Acquisition Time & 4.5438 \\
13 Spectrometer Frequency 400.13 \\
14 Spectral Width & 7211.5 \\
15 Lowest Frequency & -822.9 \\
16 Nucleus & $1 \mathrm{H}$ \\
17 Acquired Size & 32768 \\
18 Spectral Size & 65536 \\
\hline
\end{tabular}<smiles>CCOC(=O)CC(=O)C(C)[C@@H](O)c1ccc(CC)o1</smiles>

s1

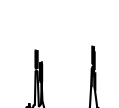

h.

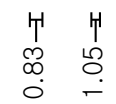

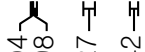

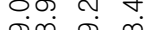$$
10
$$ 


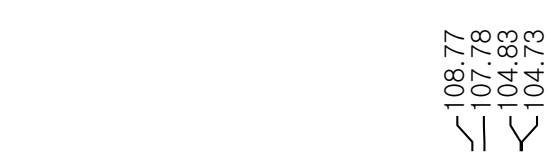

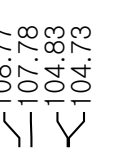<smiles>CCOC(=O)CC(=O)C(C)C(O)c1ccc(CC)o1</smiles>

s1

8 Number of Scans

9 Receiver Gain

10 Relaxation Delay

11 Pulse Width

Value

1 Origin

Varian

2 Spectrometer

vnmrs

4 Temperature

$\mathrm{cdcl} 3$

25.0

s2pul

10

oneprobe

606

60

2.0000

0.0000

12 Acquisition Time

0.8651

13 Spectrometer Frequency 150.65

14 Spectral Width

37878.8

15 Lowest Frequency

$-2332.7$

16 Nucleus

$13 \mathrm{C}$

17 Acquired Size

32768

18 Spectral Size

\author{
65536
}

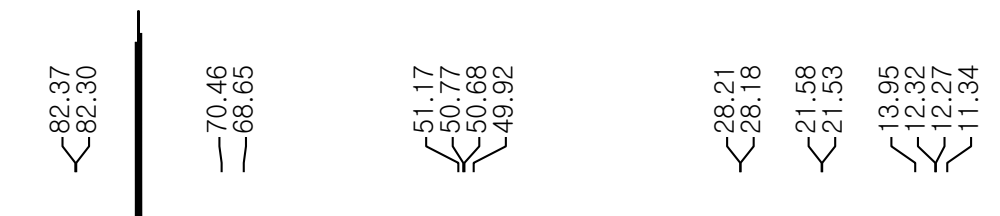

.

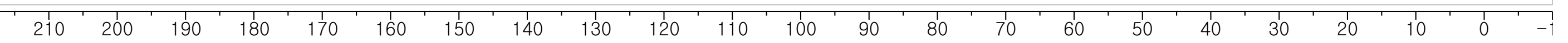




\begin{tabular}{|lll|}
\hline \multicolumn{1}{|c|}{ Parameter } & \multicolumn{1}{c|}{ Value } \\
1 & Origin & Bruker BioSpin GmbH \\
2 & Spectrometer & spect \\
3 & Solvent & $\mathrm{CDCl} 3$ \\
4 & Temperature & 297.0 \\
5 & Pulse Sequence & $\mathrm{zg} 30$ \\
6 & Experiment & $1 \mathrm{D}$ \\
7 & Probe & $5 \mathrm{~mm}$ PABBO BB/ 19F-1H/ D Z-GRD Z108618/ 0389 \\
8 & Number of Scans & 42 \\
9 & Receiver Gain & 28 \\
10 Relaxation Delay & 0.2000 \\
11 Pulse Width & 15.0000 \\
12 Acquisition Time & 4.5438 \\
13 Spectrometer Frequency & 400.13 \\
14 Spectral Width & 7211.5 \\
15 Lowest Frequency & -822.5 \\
16 Nucleus & $1 \mathrm{H}$ \\
17 Acquired Size & 32768 \\
18 Spectral Size & 65536 \\
\hline
\end{tabular}<smiles>CCCCOC(=O)CC(=O)C(C)C(=O)c1ccc(CC)o1</smiles>

19

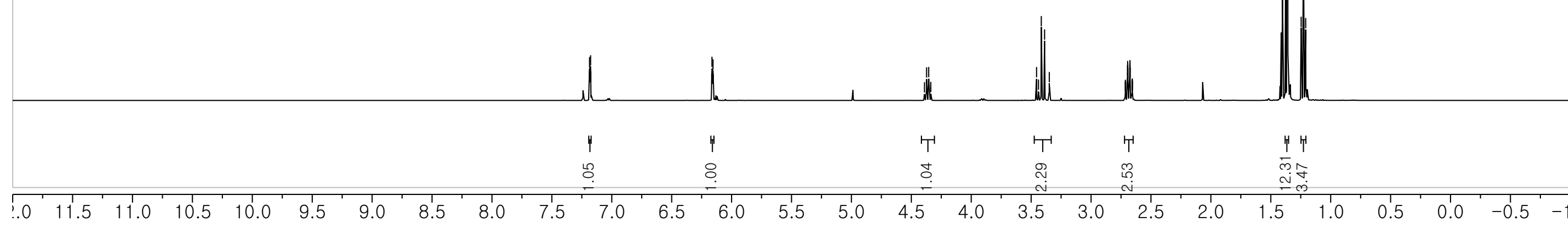




\begin{tabular}{|c|c|c|c|c|c|c|c|c|c|}
\hline $\begin{array}{l}\hat{0} \\
\dot{\infty} \\
\stackrel{\infty}{0} \\
\mid\end{array}$ & 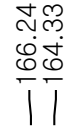 & 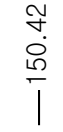 & $\begin{array}{l}\infty \\
\infty \\
\stackrel{\infty}{\infty} \\
\stackrel{\sim}{\mid}\end{array}$ & $\begin{array}{l}\stackrel{0}{0} \\
\infty \\
\stackrel{0}{\circ} \\
+1\end{array}$ & 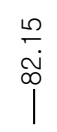 & $\begin{array}{l}\stackrel{0}{\sim} \\
\stackrel{\rho}{\circ} \\
\mid \\
\mid\end{array}$ & $\begin{array}{l}\text { os } \\
\text { o } \\
\stackrel{+}{+}\end{array}$ & $\begin{array}{l}\bar{\sigma} \\
\stackrel{\infty}{N} \\
\mid\end{array}$ & $\frac{\delta}{\sigma}$ \\
\hline
\end{tabular}

\begin{tabular}{|lll|}
\hline \multicolumn{1}{|c}{ Parameter } & \multicolumn{1}{c|}{ Value } \\
1 Origin & Varian \\
2 & Spectrometer & vnmrs \\
3 & Solvent & $c d c l 3$ \\
4 & Temperature & 25.0 \\
5 & Pulse Sequence & s2pul \\
6 Experiment & 10 \\
7 Probe & oneprobe \\
8 Number of Scans & 300 \\
9 Receiver Gain & 60 \\
10 Relaxation Delay & 2.0000 \\
11 Pulse Width & 0.0000 \\
12 Acquisition Time & 0.8651 \\
13 Spectrometer Frequency & 150.66 \\
14 Spectral Width & 37878.8 \\
15 Lowest Frequency & -2340.1 \\
16 Nucleus & $13 C$ \\
17 Acquired Size & 32768 \\
18 Spectral Size & 65536 \\
\hline
\end{tabular}<smiles>CCOC(=O)CC(=O)C(C)C(=O)c1ccc(CC)o1</smiles>

19

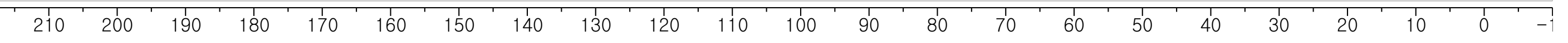




\begin{tabular}{|lll|}
\hline \multicolumn{1}{|c|}{ Parameter } & \multicolumn{1}{c|}{ Value } \\
1 & Origin & Bruker BioSpin GmbH \\
2 & Spectrometer & spect \\
3 & Solvent & CDCl3 \\
4 & Temperature & 298.0 \\
5 & Pulse Sequence & zg30 \\
6 & Experiment & 10 \\
7 & Probe & $5 \mathrm{~mm} \mathrm{PABBO} \mathrm{BB/} \mathrm{19F-1H/} \mathrm{D} \mathrm{Z-GRD} \mathrm{Z108618/} \mathrm{O389}$ \\
8 Number of Scans & 13 \\
9 Receiver Gain & 19 \\
10 Relaxation Delay & 0.2000 \\
11 Pulse Width & 15.0000 \\
12 Acquisition Time & 4.5438 \\
13 Spectrometer Frequency & 400.13 \\
14 Spectral Width & 7211.5 \\
15 Lowest Frequency & -823.2 \\
16 Nucleus & $1 \mathrm{H}$ \\
17 Acquired Size & 32768 \\
18 Spectral Size & 65536 \\
\hline
\end{tabular}<smiles>N[C@@H](C[OH+])C(O[Sb])c1ccccc1</smiles>

S3

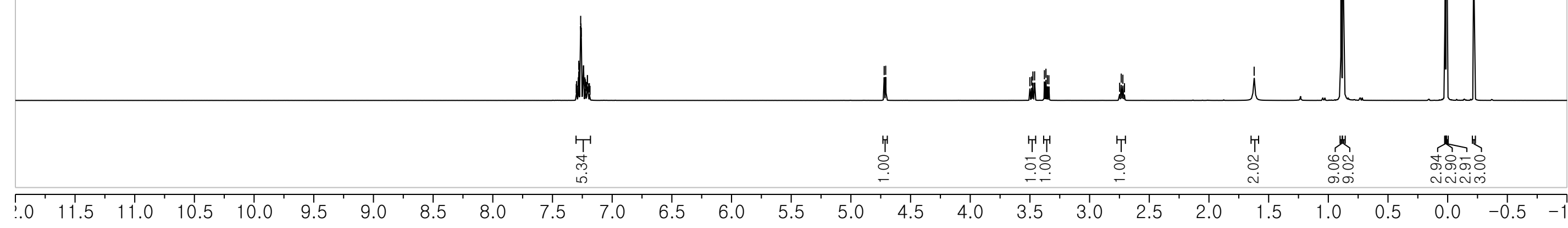




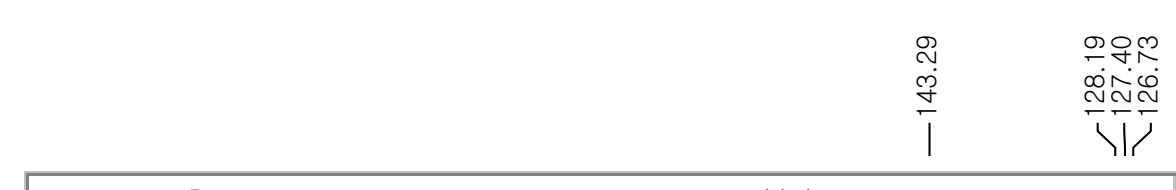

\section{Parameter}

Bruker BioSpin GmbH

\section{Origin}

2 Spectrometer

3 Solvent

4 Temperature

5 Pulse Sequence

6 Experiment

7 Probe

8 Number of Scans

9 Receiver Gain

10 Relaxation Delay

11 Pulse Width

12 Acquisition Time

14 Spectral Width $\quad 24038.5$

15 Lowest Frequency

16 Nucleus

17 Acquired Size

18 Spectral Size spect

$\mathrm{CDCl} 3$

290

10

Z116098_0402 (PA BBO 400S1 BBF-H-D-05 Z SP)

350

207

2.0000

10.0000

$-1940.4$

$13 \mathrm{C}$

32768

65536
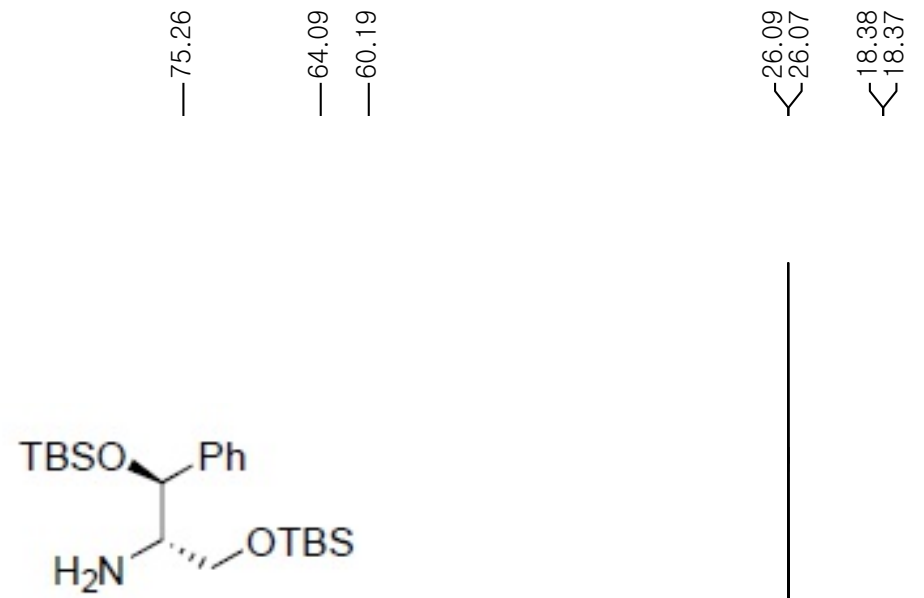

S3 


\begin{tabular}{|lll|}
\hline \multicolumn{1}{|c|}{ Parameter } & \multicolumn{1}{c|}{ Value } \\
1 & Origin & Bruker BioSpin GmbH \\
2 & Spectrometer & spect \\
3 & Solvent & $\mathrm{CDCl} 3$ \\
4 & Temperature & 298.0 \\
5 & Pulse Sequence & $\mathrm{zg} 30$ \\
6 & Experiment & $1 \mathrm{D}$ \\
7 & Probe & $5 \mathrm{~mm} \mathrm{PABBO} \mathrm{BB/} \mathrm{19F-1H/} \mathrm{D} \mathrm{Z-GRD} \mathrm{Z108618/0389}$ \\
8 & Number of Scans & 11 \\
9 & Receiver Gain & 50 \\
10 & Relaxation Delay & 0.2000 \\
11 Pulse Width & 15.0000 \\
12 Acquisition Time & 4.5438 \\
13 Spectrometer Frequency 400.13 \\
14 Spectral Width & 7211.5 \\
15 Lowest Frequency & -822.9 \\
16 Nucleus & $1 \mathrm{H}$ \\
17 Acquired Size & 32768 \\
18 Spectral Size & 65536 \\
\hline
\end{tabular}

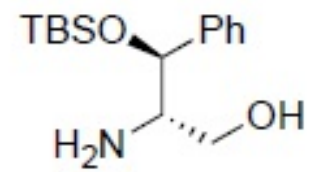

17

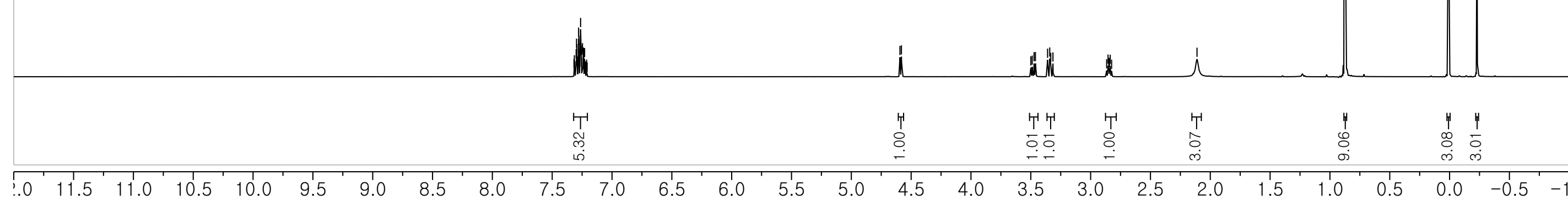




\section{Parameter}

1 Origin

2 Spectrometer

3 Solvent

4 Temperature

5 Pulse Sequence

6 Experiment

7 Probe

8 Number of Scans

9 Receiver Gain

10 Relaxation Delay

11 Pulse Width

12 Acquisition Time

13 Spectrometer Fre

14 Spectral Width

15 Lowest Frequency

16 Nucleus

17 Acquired Size

18 Spectral Size

\section{Value}

Bruker BioSpin GmbH

spect

$\mathrm{CDCl} 3$

298.0

zgpg30

10

Z116098_0402 (PA BBO 400S1 BBF-H-D-05 Z SP)

690

207

2.0000

10.0000

1.3631

1038

$-1940.2$

$13 \mathrm{C}$

32768

65536

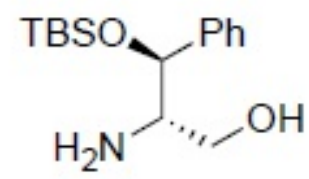

17 


\begin{tabular}{|lll|}
\hline \multicolumn{1}{|c|}{ Parameter } & \multicolumn{1}{c|}{ Value } \\
1 & Origin & Bruker BioSpin GmbH \\
2 & Spectrometer & spect \\
3 & Solvent & $\mathrm{CDCl} 3$ \\
4 & Temperature & 293.0 \\
5 & Pulse Sequence & $\mathrm{zg} 30$ \\
6 & Experiment & $1 \mathrm{D}$ \\
7 Probe & $5 \mathrm{~mm} \mathrm{PABBO} \mathrm{BB/} \mathrm{19F-1H/} \mathrm{D} \mathrm{Z-GRD} \mathrm{Z108618/} \mathrm{0389}$ \\
8 Number of Scans & 29 \\
9 Receiver Gain & 56 \\
10 Relaxation Delay & 0.2000 \\
11 Pulse Width & 15.0000 \\
12 Acquisition Time & 4.5438 \\
13 Spectrometer Frequency & 400.13 \\
14 Spectral Width & 7211.5 \\
15 Lowest Frequency & -822.9 \\
16 Nucleus & $1 \mathrm{H}$ \\
17 Acquired Size & 32768 \\
18 Spectral Size & 65536 \\
\hline
\end{tabular}<smiles>CCc1ccc(C(=O)C(C)C(=O)CC(=O)N[C@H](CO)[C@H](O[AsH3])c2ccccc2)o1</smiles>

20

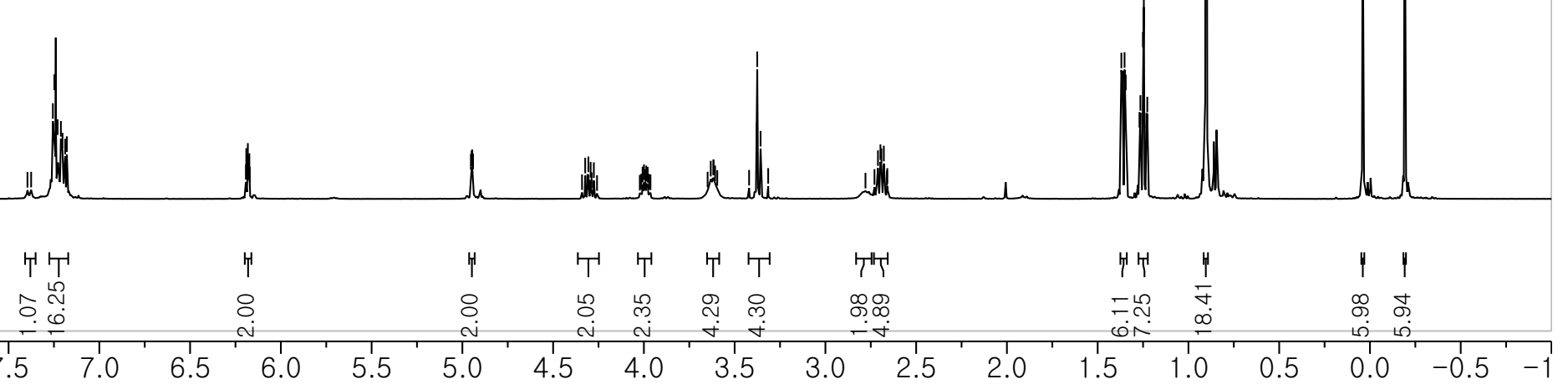


2 Spectrometer

vnmrs

3 Solvent

$\mathrm{cdcl} 3$

4 Temperature

25.0

5 Pulse Sequence

s2pul

6 Experiment

7 Probe

8 Number of Scans

oneprobe

9 Receiver Gain

300

10 Relaxation Delay

2.0000

11 Pulse Width

0.0000

12 Acquisition Time

0.8651

13 Spectrometer Frequency 150.66

14 Spectral Width $\quad 37878.8$

15 Lowest Frequency

$-2335.3$

16 Nucleus

17 Acquired Size

$13 \mathrm{C}$

18 Spectral Size<smiles>CCc1ccc(C(=O)C(C)C(=O)CC(=O)N[C@H](CO)[C@H](OS(C)(=O)=O)c2ccccc2)o1</smiles>

20$$
65536
$$

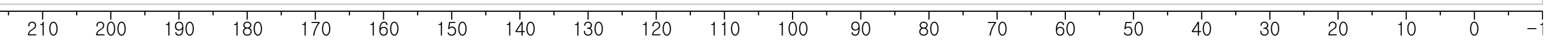




\begin{tabular}{|lll|}
\hline \multicolumn{1}{|c|}{ Parameter } & \multicolumn{1}{c|}{ Value } \\
1 & Origin & Bruker BioSpin GmbH \\
2 & Spectrometer & spect \\
3 & Solvent & $\mathrm{CDCl} 3$ \\
4 & Temperature & 298.0 \\
5 & Pulse Sequence & zg30 \\
6 & Experiment & 10 \\
7 & Probe & $5 \mathrm{~mm} \mathrm{PABBO} \mathrm{BB/} \mathrm{19F-1H/} \mathrm{D} \mathrm{Z-GRD} \mathrm{Z108618/} \mathrm{0389}$ \\
8 & Number of Scans & 21 \\
9 & Receiver Gain & 202 \\
10 Relaxation Delay & 0.2000 \\
11 Pulse Width & 15.0000 \\
12 Acquisition Time & 4.5438 \\
13 Spectrometer Frequency & 400.13 \\
14 Spectral Width & 7211.5 \\
15 Lowest Frequency & -822.8 \\
16 Nucleus & $1 \mathrm{H}$ \\
17 Acquired Size & 32768 \\
18 Spectral Size & 65536 \\
\hline
\end{tabular}<smiles>CCc1ccc(C2=C(C)C(=O)[C@@]3(O2)C(=O)N[C@](O)([C@H](O)c2ccccc2)[C@H]3O)o1</smiles><smiles>CCc1ccc(C2=C(C)C(=O)[C@]3(O2)C(=O)N[C@](O)([C@H](O)c2ccccc2)[C@H]3O)o1</smiles>

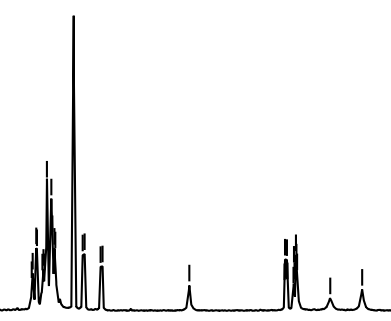

उ.




\begin{tabular}{|lll|}
\hline \multicolumn{1}{|c}{ Parameter } & \multicolumn{1}{c|}{$\begin{array}{c}\text { Value } \\
1 \text { Origin }\end{array}$} \\
2 Varian \\
3 Spectrometer & Solvent & vnmrs \\
4 & Cdcl3 \\
5 & Pulse Sequence & 25.0 \\
6 Experiment & s2pul \\
7 Probe & 10 \\
8 Number of Scans & oneprobe \\
9 Receiver Gain & 670 \\
10 Relaxation Delay & 2.0000 \\
11 Pulse Width & 0.0000 \\
12 Acquisition Time & 0.8651 \\
13 Spectrometer Frequency & 150.65 \\
14 Spectral Width & 37878.8 \\
15 Lowest Frequency & -2334.1 \\
16 Nucleus & $13 \mathrm{C}$ \\
17 Acquired Size & 32768 \\
18 Spectral Size & 65536 \\
\hline
\end{tabular}

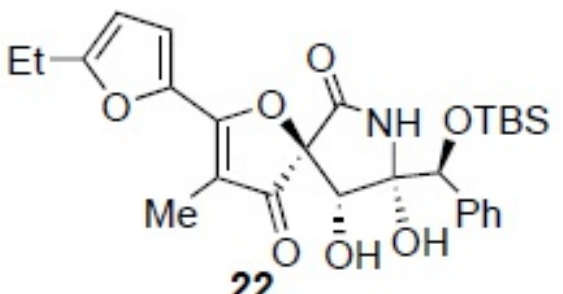<smiles>CCc1ccc(C2=C(C)C(=O)[C@]3(O2)C(=O)N[C@](O)([C@H](O)c2ccccc2)[C@H]3O)o1</smiles> 


\begin{tabular}{|lll|}
\hline \multicolumn{1}{|c|}{ Parameter } & \multicolumn{1}{c|}{ Value } \\
1 & Origin & Bruker BioSpin GmbH \\
2 & Spectrometer & spect \\
3 & Solvent & $\mathrm{CDCl} 3$ \\
4 & Temperature & 298.0 \\
5 & Pulse Sequence & $\mathrm{zg} 30$ \\
6 & Experiment & $1 \mathrm{D}$ \\
7 & Probe & $5 \mathrm{~mm} \mathrm{PABBO} \mathrm{BB/}$ 19F-1H/ D Z-GRD Z108618/ 0389 \\
8 & Number of Scans & 22 \\
9 Receiver Gain & 202 \\
10 Relaxation Delay & 0.2000 \\
11 Pulse Width & 15.0000 \\
12 Acquisition Time & 4.5438 \\
13 Spectrometer Frequency & 400.13 \\
14 Spectral Width & 7211.5 \\
15 Lowest Frequency & -822.9 \\
16 Nucleus & $1 \mathrm{H}$ \\
17 Acquired Size & 32768 \\
18 Spectral Size & 65536 \\
\hline
\end{tabular}

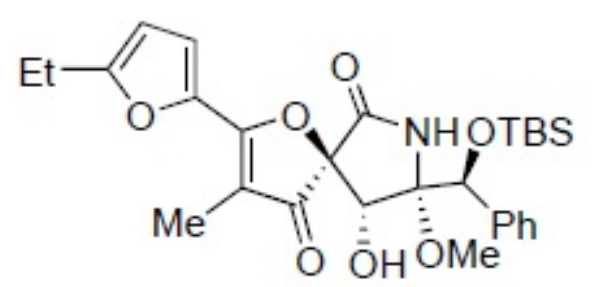

30

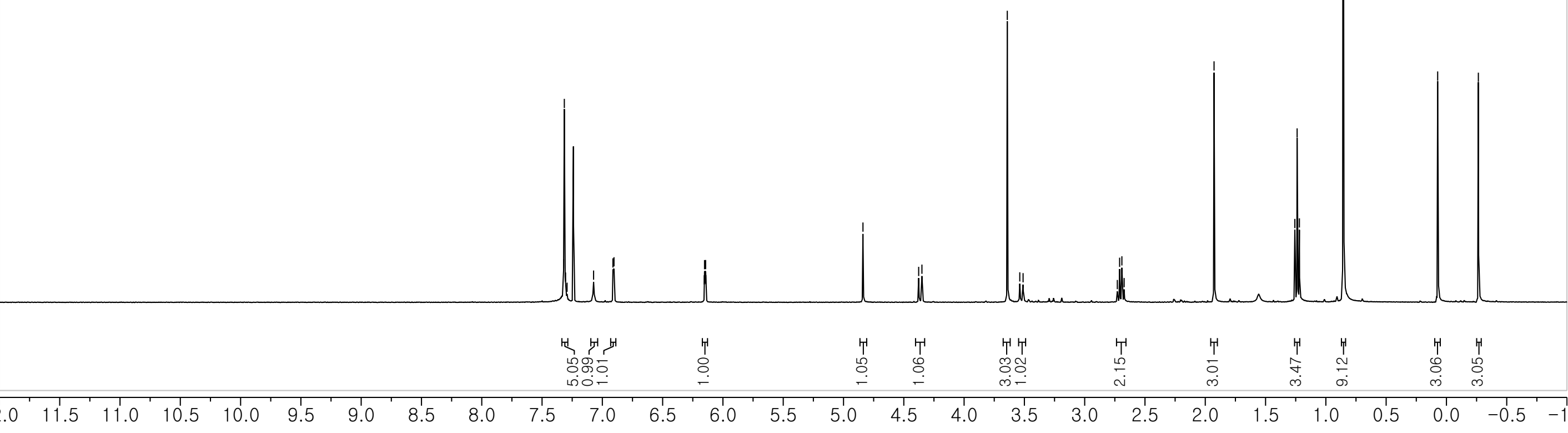




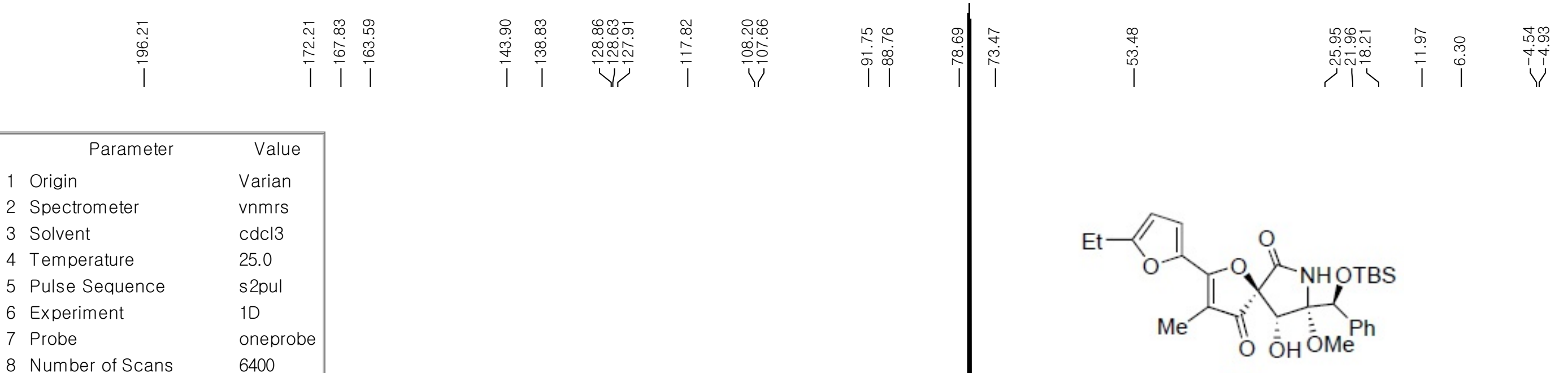

\begin{tabular}{|ll|}
8 Rumber of Scans & 6400 \\
10 Relaxation Delay & 60 \\
11 Pulse Width & 2.0000 \\
12 Acquisition Time & 0.0000 \\
13 Spectrometer Frequency & 150.66 \\
14 Spectral Width & 37878.8 \\
15 Lowest Frequency & -2333.2 \\
16 Nucleus & $13 C$ \\
17 Acquired Size & 32768 \\
18 Spectral Size & 65536 \\
\hline
\end{tabular}

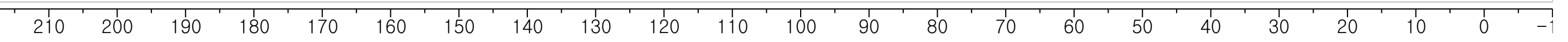


1 Origin

Parameter

2 Spectrometer

3 Solvent

4 Temperature

5 Pulse Sequence

6 Experiment

7 Probe

8 Number of Scans

9 Receiver Gain

10 Relaxation Delay

11 Pulse Width

12 Acquisition Time

15 Lowest Frequency $\quad-801.3$

16 Nucleus

17 Acquired Size

18 Spectral Size
-801.3
$1 \mathrm{H}$

Bruker BioSpin GmbH

spect

298.0

zg30

10 17

0.2000

15.0000

400.13

32768

65536

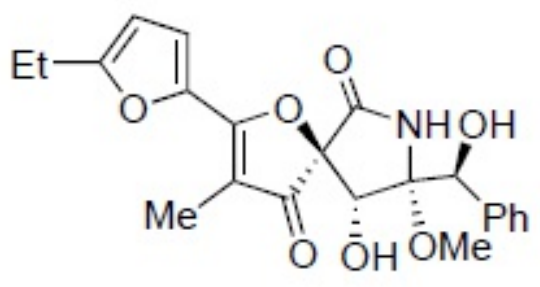

31

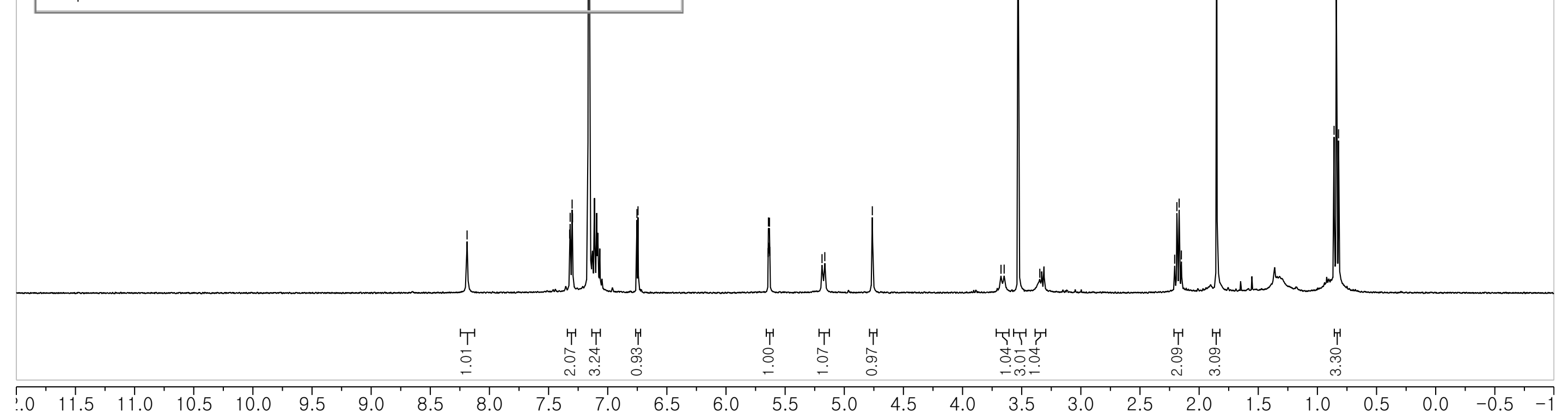


1 Origin

Bruker BioSpin $\mathrm{GmbH}$

2 Spectrometer

spect

3 Solvent

$\mathrm{CDCl} 3$

298.0

4 Temperature

5 Pulse Sequence

zg30

6 Experiment

7 Probe

8 Number of Scans

9 Receiver Gain

10 Relaxation Delay

11 Pulse Width

12 Acquisition Time

10

5 mm PABBO BB/ 19F-1H/ D Z-GRD Z 108618/ 0389 21

202

0.2000

15.0000

4.5438

13 Spectrometer Frequency 400.13

14 Spectral Width

7211.5

15 Lowest Frequency

16 Nucleus

$-822.9$

17 Acquired Size

18 Spectral Size
$1 \mathrm{H}$

32768

65536

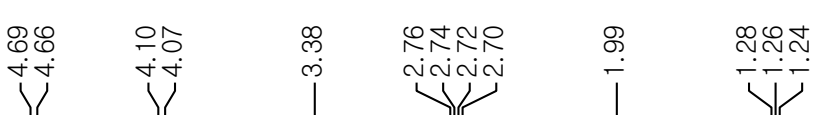

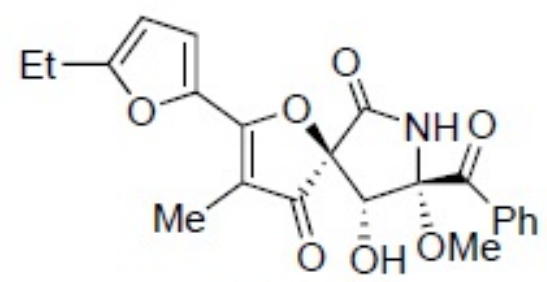

(-)-FD-838 (5)

$$
\frac{10}{.0}
$$

\begin{tabular}{|c|c|}
\hline $\begin{array}{l}\stackrel{H}{1} \\
\stackrel{\sim}{O} \\
\stackrel{\sim}{\sim}\end{array}$ & 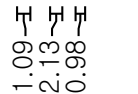 \\
\hline
\end{tabular}

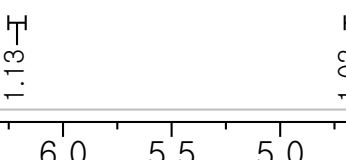

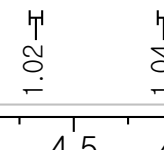

$\frac{T_{1}^{+}}{T^{\prime}}$

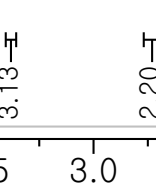

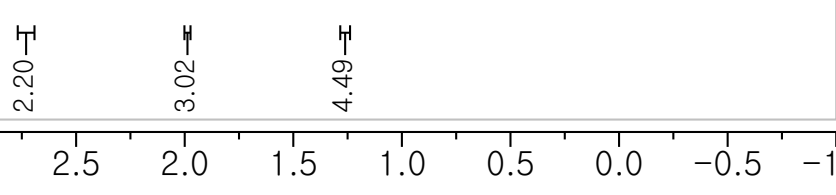




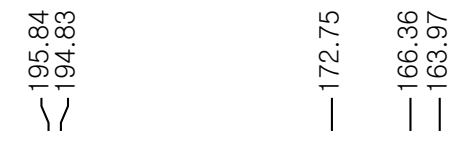

Parameter

\section{Value}

1 Origin

Varian

2 Spectrometer

3 Solvent

vnmrs

4 Temperature

5 Pulse Sequence

6 Experiment

7 Probe

8 Number of Scans

9 Receiver Gain

10 Relaxation Delay

11 Pulse Width

12 Acquisition Time

14 Spectral Width

15 Lowest Frequency

16 Nucleus

17 Acquired Size

18 Spectral Size

oneprobe

606

2.0000

0.0000

0.8651

37878.8

$-2332.0$

$13 \mathrm{C}$

32768

65536

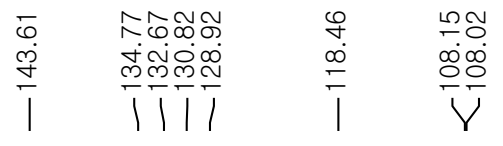

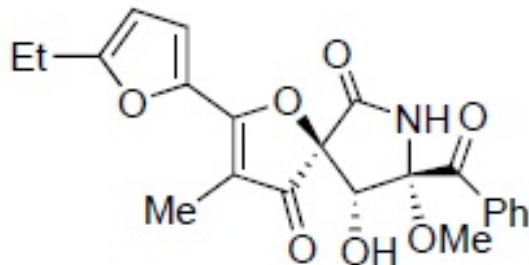

(-)-FD-838 (5)

\begin{tabular}{lllllllllllllllllllllllll}
\hline 1 & 110 & 200 & 190 & 180 & 170 & 160 & 150 & 140 & 130 & 120 & 110 & 100 & 90 & 80 & 70 & 60 & 50 & 40 & 30 & 20 & 10 & 0 & -1
\end{tabular}




\begin{tabular}{|lll|}
\hline \multicolumn{2}{|c|}{ Parameter } & \multicolumn{1}{c|}{ Value } \\
1 & Origin & Bruker BioSpin GmbH \\
2 & Spectrometer & spect \\
3 & Solvent & $\mathrm{CDCl} 3$ \\
4 & Temperature & 298.1 \\
5 & Pulse Sequence & $\mathrm{zg} 30$ \\
6 & Experiment & 10 \\
7 & Probe & $5 \mathrm{~mm}$ PABBO BB/ 19F-1H/ D Z-GRD Z108618/ O389 \\
8 Number of Scans & 38 \\
9 & Receiver Gain & 36 \\
10 Relaxation Delay & 0.2000 \\
11 Pulse Width & 15.0000 \\
12 Acquisition Time & 4.5438 \\
13 Spectrometer Frequency & 400.13 \\
14 Spectral Width & 7211.5 \\
15 Lowest Frequency & -822.6 \\
16 Nucleus & $1 \mathrm{H}$ \\
17 Acquired Size & 32768 \\
18 Spectral Size & 65536 \\
\hline
\end{tabular}<smiles>CC/C=C/CC[C@@H](O)[C@@H](C)C(=O)CC(=O)OCCC</smiles> 


\begin{tabular}{|lll|}
\hline \multicolumn{1}{|c}{ Parameter } & \multicolumn{1}{c|}{ Value } \\
1 & Origin & Varian \\
2 & Spectrometer & vnmrs \\
3 & Solvent & cdcl3 \\
4 & Temperature & 25.0 \\
5 & Pulse Sequence & s2pul \\
6 Experiment & 10 \\
7 Probe & oneprobe \\
8 Number of Scans & 606 \\
9 Receiver Gain & 60 \\
10 Relaxation Delay & 2.0000 \\
11 Pulse Width & 0.0000 \\
12 Acquisition Time & 0.8651 \\
13 Spectrometer Frequency & 150.65 \\
14 Spectral Width & 37878.8 \\
15 Lowest Frequency & -2335.6 \\
16 Nucleus & $13 \mathrm{C}$ \\
17 Acquired Size & 32768 \\
18 Spectral Size & 65536 \\
\hline
\end{tabular}<smiles>CC/C=C/CC[C@H](O)[C@H](C)C(=O)CC(=O)OCC</smiles>
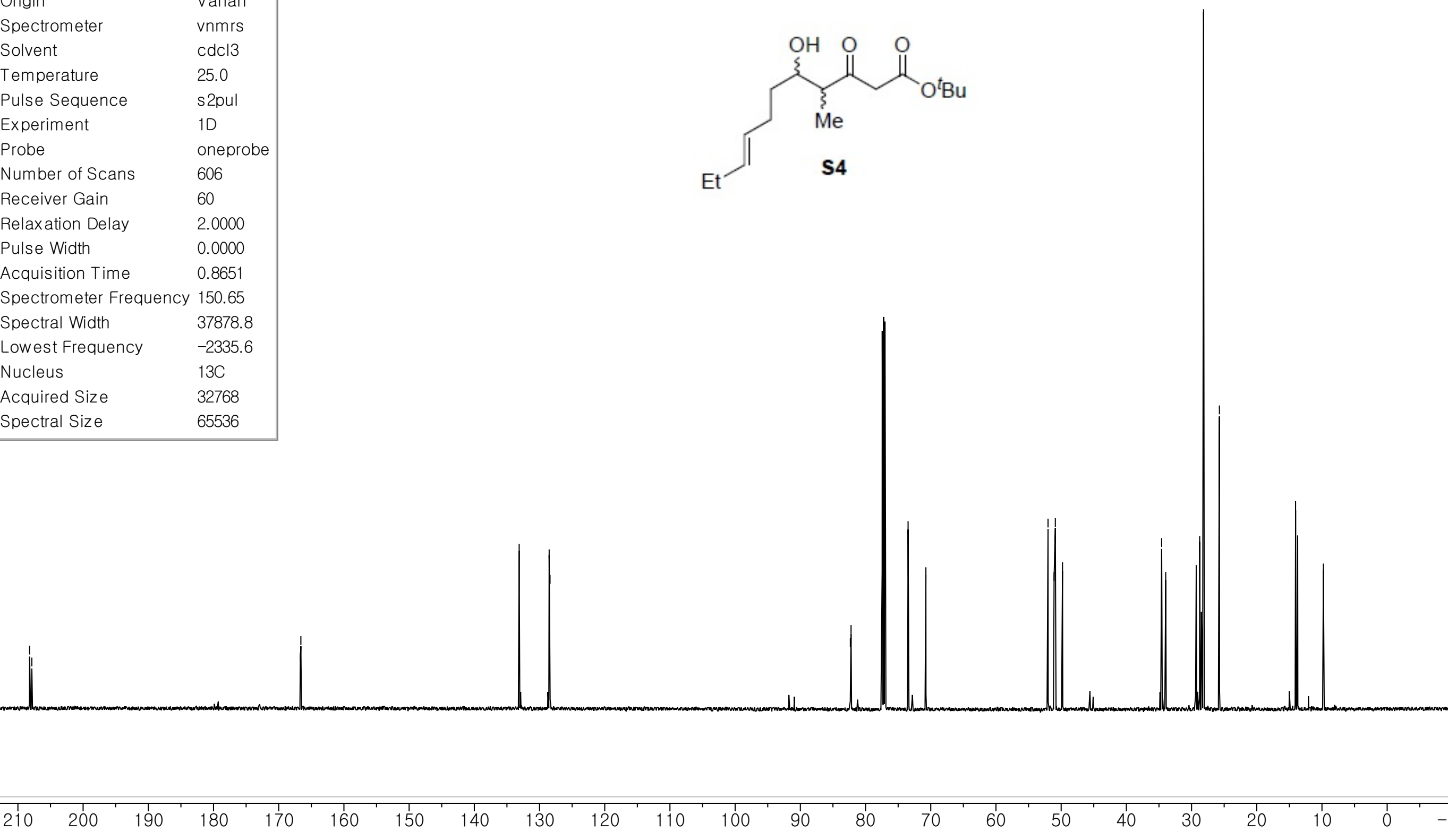


\begin{tabular}{|lll|}
\hline \multicolumn{1}{|c|}{ Parameter } & \multicolumn{1}{c|}{ Value } \\
1 & Origin & Bruker BioSpin GmbH \\
2 & Spectrometer & spect \\
3 & Solvent & $\mathrm{CDCl} 3$ \\
4 & Temperature & 298.2 \\
5 & Pulse Sequence & zg30 \\
6 & Experiment & 10 \\
7 & Probe & $5 \mathrm{~mm} \mathrm{PABBO} \mathrm{BB/} \mathrm{19F-1H/} \mathrm{D} \mathrm{Z-GRD} \mathrm{Z108618/} \mathrm{0389}$ \\
8 & Number of Scans & 31 \\
9 & Receiver Gain & 32 \\
10 Relaxation Delay & 0.2000 \\
11 Pulse Width & 15.0000 \\
12 Acquisition Time & 4.5438 \\
13 Spectrometer Frequency & 400.13 \\
14 Spectral Width & 7211.5 \\
15 Lowest Frequency & -823.4 \\
16 Nucleus & $1 \mathrm{H}$ \\
17 Acquired Size & 32768 \\
18 Spectral Size & 65536 \\
\hline
\end{tabular}<smiles>CC/C=C/CCC(=O)C(C)C(=O)CC(=O)OCCC</smiles> 


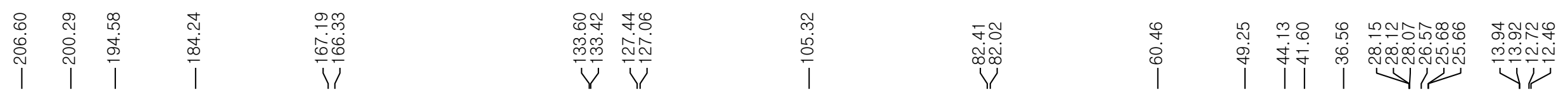

\begin{tabular}{|lll|}
\hline \multicolumn{1}{|c}{ Parameter } & \multicolumn{1}{c|}{ Value } \\
1 Origin & Varian \\
2 & Spectrometer & vnmrs \\
3 & Solvent & $c d c l 3$ \\
4 & Temperature & 25.0 \\
5 & Pulse Sequence & s2pul \\
6 Experiment & 10 \\
7 Probe & oneprobe \\
8 Number of Scans & 256 \\
9 Receiver Gain & 60 \\
10 Relaxation Delay & 2.0000 \\
11 Pulse Width & 0.0000 \\
12 Acquisition Time & 0.8651 \\
13 Spectrometer Frequency & 150.66 \\
14 Spectral Width & 37878.8 \\
15 Lowest Frequency & -2334.5 \\
16 Nucleus & $13 \mathrm{C}$ \\
17 Acquired Size & 32768 \\
18 Spectral Size & 65536 \\
\hline
\end{tabular}<smiles>CC/C=C/CCC(=O)C(C)C(=O)CC(=O)OCC</smiles>

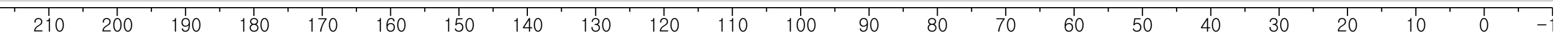




\begin{tabular}{|lll|}
\hline \multicolumn{1}{|c|}{ Parameter } & \multicolumn{1}{c|}{ Value } \\
1 & Origin & Bruker BioSpin GmbH \\
2 & Spectrometer & spect \\
3 & Solvent & CDCl3 \\
4 & Temperature & 299.7 \\
5 & Pulse Sequence & zg30 \\
6 & Experiment & 10 \\
7 Probe & $5 \mathrm{~mm} \mathrm{PABBO} \mathrm{BB/} \mathrm{19F-1H/} \mathrm{D} \mathrm{Z-GRD} \mathrm{Z108618/} \mathrm{0389}$ \\
8 Number of Scans & 24 \\
9 Receiver Gain & 32 \\
10 Relaxation Delay & 0.2000 \\
11 Pulse Width & 15.0000 \\
12 Acquisition Time & 4.5438 \\
13 Spectrometer Frequency & 400.13 \\
14 Spectral Width & 7211.5 \\
15 Lowest Frequency & -822.6 \\
16 Nucleus & $1 \mathrm{H}$ \\
17 Acquired Size & 32768 \\
18 Spectral Size & 65536 \\
\hline
\end{tabular}<smiles>CC/C=C/CCC(=O)C(C)C(=O)CC(=O)N[C@H](CO)[C@H](O)c1ccccc1</smiles>

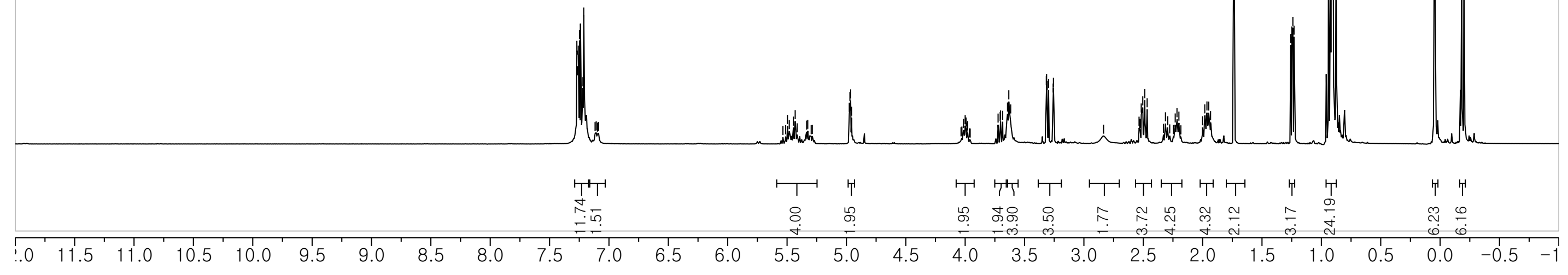




\begin{tabular}{|lll|}
\hline \multicolumn{1}{|c}{ Parameter } & \multicolumn{1}{c|}{ Value } \\
1 & Origin & Varian \\
2 & Spectrometer & vnmrs \\
3 & Solvent & cdcl3 \\
4 & Temperature & 25.0 \\
5 & Pulse Sequence & s2pul \\
6 Experiment & 10 \\
7 Probe & oneprobe \\
8 Number of Scans & 256 \\
9 Receiver Gain & 60 \\
10 Relaxation Delay & 2.0000 \\
11 Pulse Width & 0.0000 \\
12 Acquisition Time & 0.8651 \\
13 Spectrometer Frequency & 150.66 \\
14 Spectral Width & 37878.8 \\
15 Lowest Frequency & -2350.1 \\
16 Nucleus & $13 \mathrm{C}$ \\
17 Acquired Size & 32768 \\
18 Spectral Size & 65536 \\
\hline
\end{tabular}<smiles>CC/C=C/CCC(=O)C(C)C(=O)CC(=O)N[C@H](CO)[C@H](O)c1ccccc1</smiles>
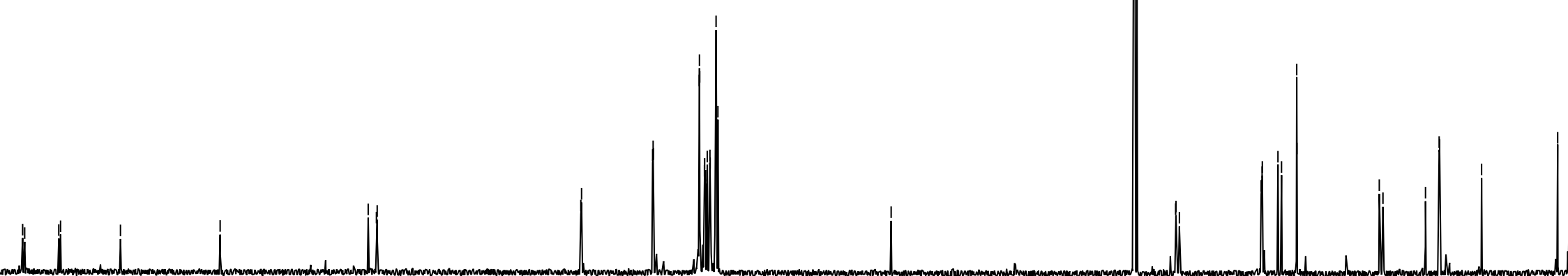

$210 \quad 200 \quad 190 \quad 180 \quad 170$




$$
\begin{array}{ll}
\overline{5} & \infty \\
\multirow{2}{0}{} & \infty \\
\hline & \infty
\end{array}
$$

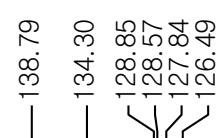

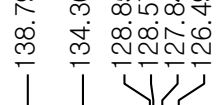

Parameter

Value

1 Origin

Varian

2 Spectrometer

vnmrs

3 Solvent

$\mathrm{cdcl} 3$

25.0

s2pul

5 Pulse Sequence

10

6 Experiment

oneprobe

606

8 Number of Scans

60

2.0000

10 Relaxation Delay

11 Pulse Width

0.0000

0.8651

12 Acquisition Time

150.66

14 Spectral Width $\quad 37878.8$

15 Lowest Frequency

$-2333.5$

16 Nucleus

17 Acquired Size

$13 \mathrm{C}$

32768

65536

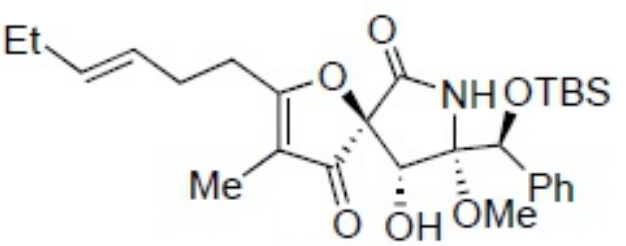

36

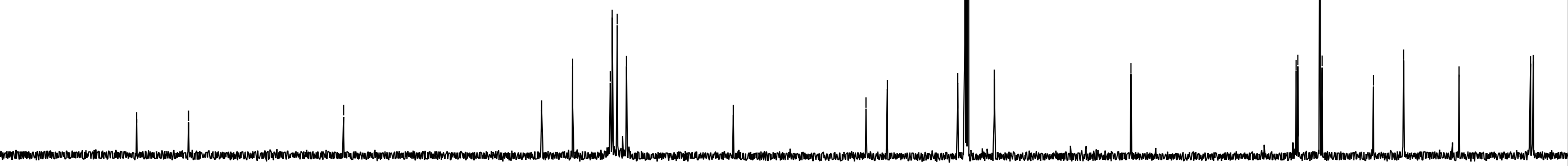

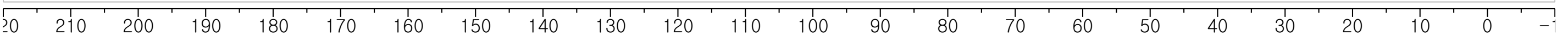




\begin{tabular}{|lll|}
\hline \multicolumn{1}{|c|}{ Parameter } & \multicolumn{1}{c|}{ Value } \\
1 & Origin & Bruker BioSpin GmbH \\
2 & Spectrometer & spect \\
3 & Solvent & CD2Cl2 \\
4 & Temperature & 298.0 \\
5 & Pulse Sequence & zg30 \\
6 & Experiment & 10 \\
7 & Probe & $5 \mathrm{~mm} \mathrm{PABBO} \mathrm{BB/}$ 19F-1H/ D Z-GRD Z108618/ 0389 \\
8 & Number of Scans & 28 \\
9 & Receiver Gain & 202 \\
10 Relaxation Delay & 0.2000 \\
11 Pulse Width & 15.0000 \\
12 Acquisition Time & 4.5438 \\
13 Spectrometer Frequency & 400.13 \\
14 Spectral Width & 7211.5 \\
15 Lowest Frequency & -820.1 \\
16 Nucleus & $1 \mathrm{H}$ \\
17 Acquired Size & 32768 \\
18 Spectral Size & 65536 \\
\hline
\end{tabular}

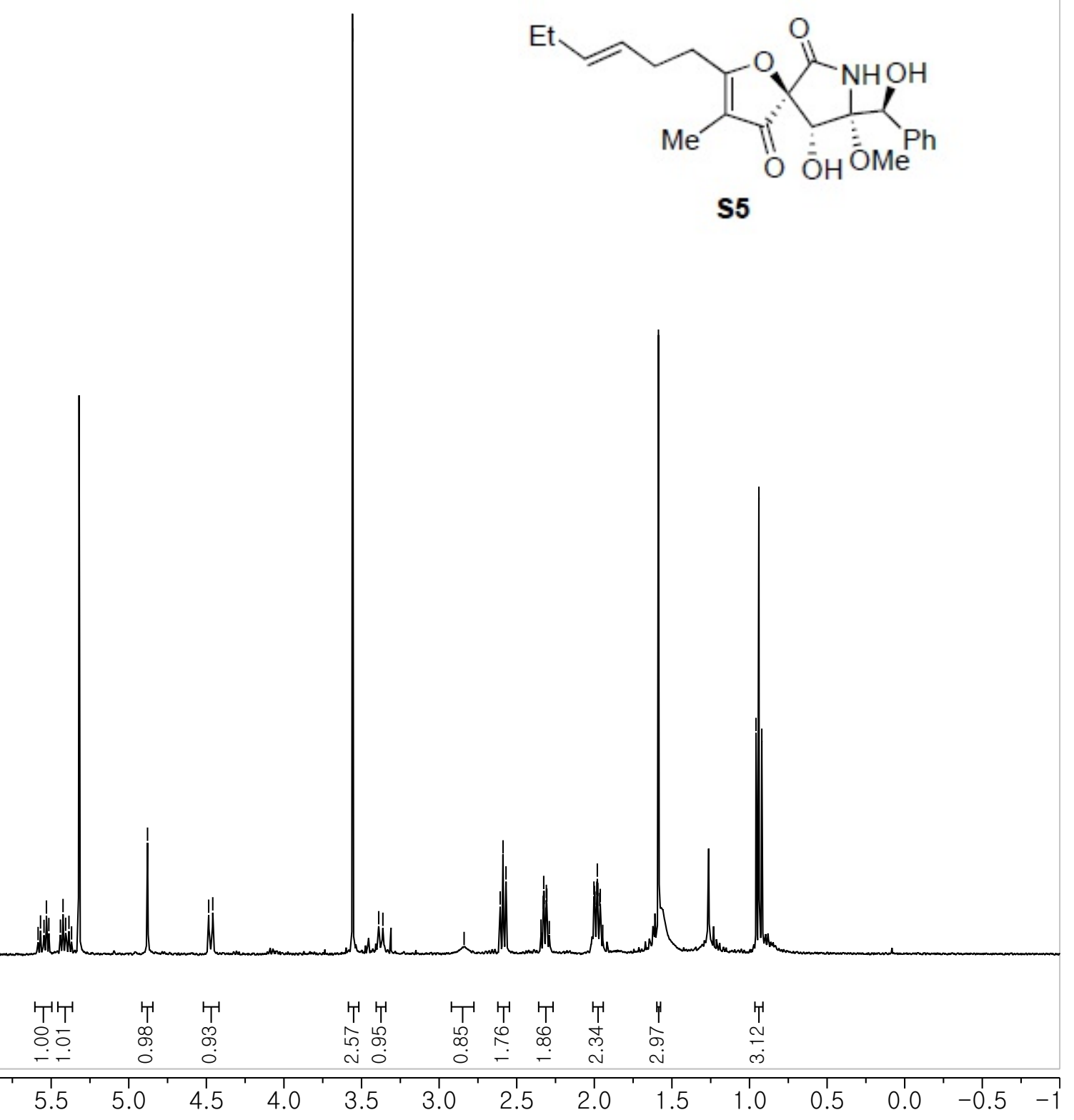




\begin{tabular}{|c|c|c|c|c|}
\hline 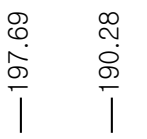 & $\begin{array}{l}\stackrel{0}{\rho} \\
\hat{0} \\
\end{array}$ & 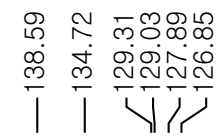 & $\frac{m}{m}$ & 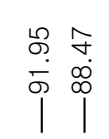 \\
\hline
\end{tabular}

\begin{tabular}{|lll|}
\hline \multicolumn{1}{|c|}{ Parameter } & \multicolumn{1}{c|}{ Value } \\
1 & Origin & Varian \\
2 & Spectrometer & vnmrs \\
3 & Solvent & cd2cl2 \\
4 & Temperature & 25.0 \\
5 & Pulse Sequence & s2pul \\
6 & Experiment & 10 \\
7 & Probe & oneprobe \\
8 & Number of Scans & 555 \\
9 & Receiver Gain & 60 \\
10 Relaxation Delay & 2.0000 \\
11 Pulse Width & 0.0000 \\
12 Acquisition Time & 0.8651 \\
13 Spectrometer Frequency 150.65 \\
14 Spectral Width & 37878.8 \\
15 Lowest Frequency & -2275.7 \\
16 Nucleus & $13 \mathrm{C}$ \\
17 Acquired Size & 32768 \\
18 Spectral Size & 65536 \\
\hline
\end{tabular}<smiles>CC/C=C/CCC1=C(C)C(=O)[C@]2(O1)C(=O)N[C@](O)([C@H](O)c1ccccc1)[C@H]2O</smiles>

S5 


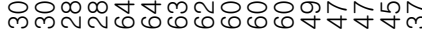

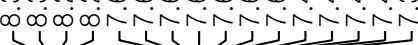

Parameter

1 Origin

2 Spectrometer

3 Solvent

4 Temperature

5 Pulse Sequence

6 Experiment

7 Probe

8 Number of Scans

9 Receiver Gain

10 Relaxation Delay

11 Pulse Width

12 Acquisition Time

15 Lowest Frequency

16 Nucleus

17 Acquired Size

18 Spectral Size
13 Spectrometer Frequency 400.13

14 Spectral Width $\quad 7211.5$
Bruker BioSpin $\mathrm{GmbH}$

spect

$\mathrm{CDCl} 3$

298.0

zg30

10

5 mm PABBO BB/ 19F-1H/ D Z-GRD Z108618/ 0389

25

202

0.2000

15.0000

4.5438

7211.5

$1 \mathrm{H}$

32768

65536

\section{Value}

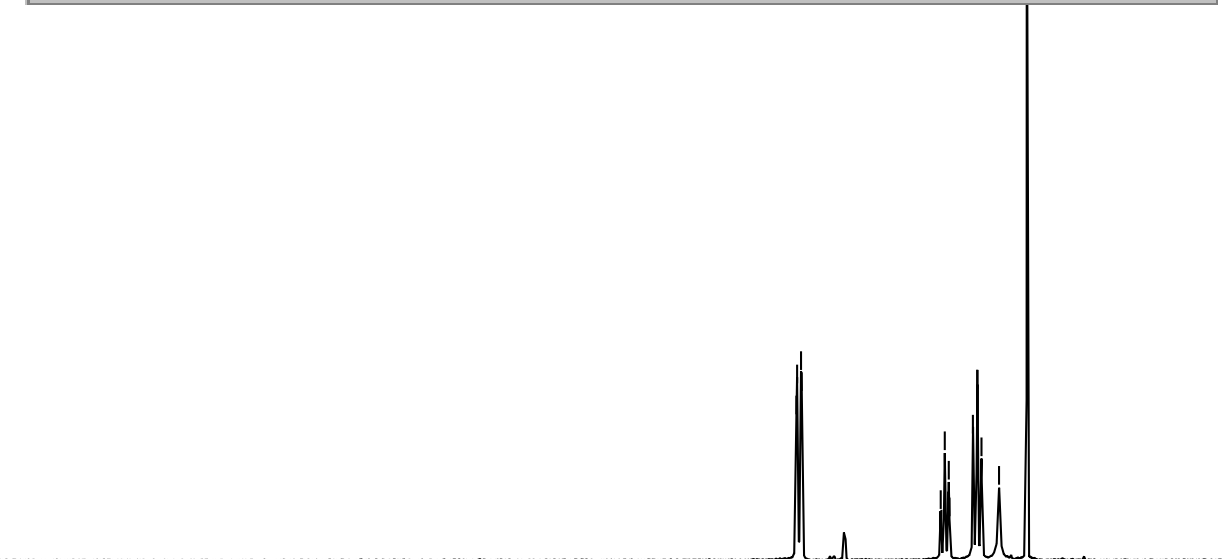

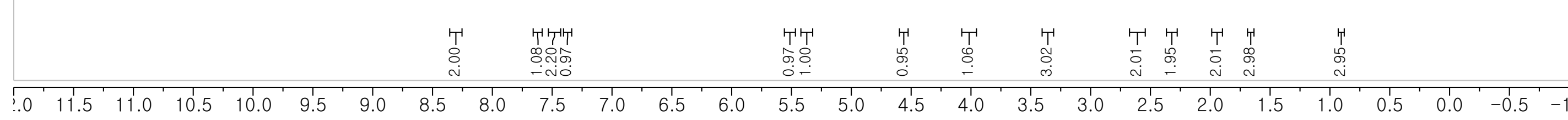




\begin{tabular}{lll}
\hline \multicolumn{1}{c}{ Parameter } & \multicolumn{1}{c}{ Value } \\
1 Origin & Varian \\
2 Spectrometer & vnmrs \\
3 Sdcl3 \\
4 Temperature & 25.0 \\
5 Pulse Sequence & s2pul \\
6 Experiment & 10 \\
7 Probe & oneprobe \\
8 Number of Scans & 606 \\
9 Receiver Gain & 60 \\
10 Relaxation Delay & 2.0000 \\
11 Pulse Width & 0.0000 \\
12 Acquisition Time & 0.8651 \\
13 Spectrometer Frequency & 150.65 \\
14 Spectral Width & 37878.8 \\
15 Lowest Frequency & -2332.3 \\
16 Nucleus & $13 \mathrm{C}$ \\
17 Acquired Size & 32768 \\
18 Spectral Size & 65536
\end{tabular}

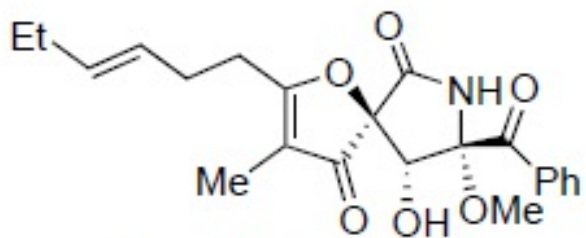

(-)-cephalimysin A (6)

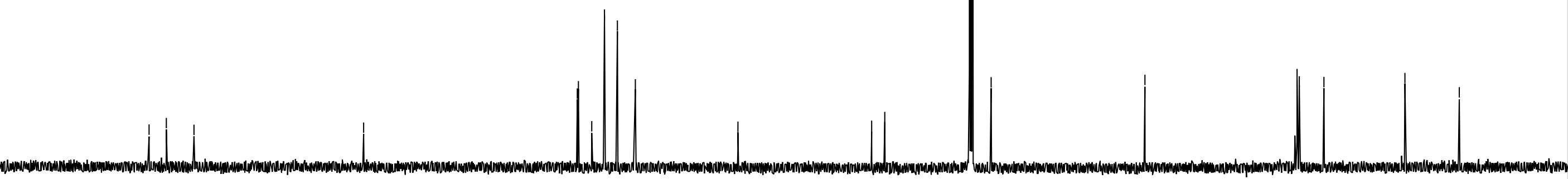

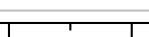

120

$110 \quad 100$

$90 \quad 80$

